\title{
MULTIPLICITIES IN SELMER GROUPS AND ROOT NUMBERS FOR ARTIN TWISTS
}

\author{
SOMNATH JHA, TATHAGATA MANDAL AND SUDHANSHU SHEKHAR
}

\begin{abstract}
Let $K / F$ be a finite Galois extension of number fields and $\sigma$ be an absolutely irreducible, self-dual representation of $\operatorname{Gal}(K / F)$. Let $p$ be an odd prime and consider two elliptic curves $E_{1}, E_{2}$ with good, ordinary reduction at primes above $p$ and equivalent mod- $p$ Galois representations. In this article, we study the variation of the parity of the multiplicities of $\sigma$ in the representation space associated to the $p^{\infty}$-Selmer group of $E_{i}$ over $K$. We also compare the root numbers for the twist of $E_{i} / F$ by $\sigma$ and show that the $p$-parity conjecture holds for the twist of $E_{1} / F$ by $\sigma$ if and only if it holds for the twist of $E_{2} / F$ by $\sigma$. We also express Mazur-Rubin-Nekováŕ's arithmetic local constants in terms of certain local Iwasawa invariants.
\end{abstract}

\section{INTRODUCTION}

In this article, we study the variation of the $p$-parity conjecture for Artin twists of two elliptic curves over certain number field, that have equivalent mod- $p$ Galois representations.

Let $E$ be an elliptic curve over a number field $K$. Let $W(E / K) \in\{ \pm 1\}$ be the (global) root number (§3) given by the sign of the expected functional equation of the complex $L$-function $L(E / K, s)$. Given a rational prime $p$ and a number field $K$, recall the definition of $p^{\infty}$-Selmer group of $E$ over $K$ :

$$
0 \longrightarrow S_{p}(E / K) \longrightarrow \mathrm{H}^{1}\left(K, E\left[p^{\infty}\right]\right) \longrightarrow \prod_{\text {all prime } u \text { in } K} \mathrm{H}^{1}\left(K_{u}, E\right),
$$

with $E\left[p^{\infty}\right]:=\cup_{n \geq 1} E\left[p^{n}\right](\bar{K})$. The Pontryagin dual $S_{p}(E / K)^{\vee}:=\operatorname{Hom}_{\text {cont }}\left(S_{p}(E / K), \mathbb{Q}_{p} / \mathbb{Z}_{p}\right)$ is a finitely generated $\mathbb{Z}_{p}$ module and we define $s_{p}:=\operatorname{rank}_{\mathbb{Z}_{p}} S_{p}(E / K)^{\vee}=\operatorname{dim}_{\mathbb{Q}_{p}} S_{p}(E / K)^{\vee} \otimes_{\mathbb{Z}_{p}} \mathbb{Q}_{p}$. Then the $p$-parity conjecture states that $W(E / K)=(-1)^{s_{p}}$.

The $p$-parity conjecture is known for elliptic curves defined over many number fields, due to works of many authors including Dokchitser-Dokchitser, Monsky, Mazur-Rubin, Nekovář etc. (cf. [DD09]). However for an arbitrary number field $F$, the $p$-parity conjecture is not established yet. Now, let $K / F$ be a finite Galois extension of number fields. Then the complex $L$-function $L(E / K, s)$ can be expressed as a product of $L(E / F, \sigma, s)$, where $\sigma$ varies over the irreducible Artin representations of $\operatorname{Gal}(K / F)$, each with multiplicity $\operatorname{deg}(\sigma)$. A variant of the Birch and Swinnerton-Dyer (BSD) conjecture, relates for every such $\sigma$, the multiplicity of $\sigma$ in $E(K) \otimes_{\mathbb{Z}} \mathbb{C}$ with $\operatorname{ord}_{s=1} L(E / F, \sigma, s)$ (see [Gre10, §4]). Thus one naturally considers the $p$-parity conjecture for the twists of $E / F$ by $\sigma$; relating the multiplicity of the

Key words and phrases. elliptic curves, p-parity conjecture, Artin twist, Selmer groups, root numbers, congruence of elliptic curves, arithmetic local constants.

2020 MSC classification: Primary: 11G05, 11R23, 11G40, 11F33 Secondary: 11G07 . 
Artin representations in the twisted $p^{\infty}$-Selmer groups to the root number of the twisted $L$-functions. We need a couple of definitions to state it precisely.

Fixing an embedding of $\overline{\mathbb{Q}}$ into $\overline{\mathbb{Q}}_{p}$, an Artin representation $\sigma$ of $\Delta:=\mathrm{Gal}(K / F)$ can be viewed as $\sigma: \Delta \rightarrow \mathrm{GL}_{n}(\overline{\mathbb{Q}}) \hookrightarrow \mathrm{GL}_{n}\left(\overline{\mathbb{Q}}_{p}\right)$. For an irreducible representation $\sigma: \Delta \rightarrow \mathrm{GL}_{n}\left(\overline{\mathbb{Q}}_{p}\right)$, define $s_{p}(E, \sigma)$ to be the multiplicity of $\sigma$ in the $\overline{\mathbb{Q}}_{p}$ representation space $\operatorname{Hom}_{\text {cont }}\left(S_{p}(E / K), \mathbb{Q}_{p} / \mathbb{Z}_{p}\right) \otimes_{\mathbb{Z}_{p}} \overline{\mathbb{Q}}_{p}$. On the other hand, for any self-dual irreducible (Artin) representation $\sigma$ of $\Delta=\operatorname{Gal}(K / F)$, the (global) root number $W(E / F, \sigma) \in\{ \pm 1\}$ is the sign of the expected functional equation of $L(E / F, \sigma, s)$ i.e. $W(E / F, \sigma)=(-1)^{\operatorname{ord}_{s=1} L(E / F, \sigma, s)}$ (possibly conjectural). However, in the above setting, there is a precise definition of $W(E / F, \sigma)$ due to Deligne [Del73], which does not depend on any conjecture.

The twisted $p$-parity conjecture states that for any elliptic curve $E$ defined over $F$ and any self-dual, irreducible representation $\sigma$ of $\Delta$,

$$
W(E / F, \sigma)=(-1)^{s_{p}(E / F, \sigma)} .
$$

We refer to [DD09, Theorem 1.3], where this twisted $p$-parity conjecture is proved for certain number fields $K$, under some hypotheses on $E$.

Let $\mu_{p}$ denote the group of $p$-th roots of unity. For any number field or an $\ell$-adic field $L$, we will denote by $L_{\text {сус }}$ the cyclotomic $\mathbb{Z}_{p}$-extension of $L$. The following is the main theorem (Theorem 3.5) of this paper; it studies the variation of $p$-parity conjecture of an Artin twist of two congruent elliptic curves by comparing the ratio of the root numbers of the twists with the ratio of the multiplicities of the twists in the Selmer groups.

Theorem 0.1 (Theorem 3.5). Let $p$ be an odd prime and $k$ be a number field such that all primes $v \mid p$ of $k$ are ramified in $k\left(\mu_{p}\right) / k$. Let $E_{1}, E_{2}$ be two elliptic curves over $k$ with good, ordinary reduction at all the primes $v \mid p$. We assume $E_{1}[p] \cong E_{2}[p]$ as $G_{k}$-modules. Consider number fields $k \subset F \subset K$ with $K \cap F_{\text {cyc }}=F$. Let $\sigma: \Delta=\operatorname{Gal}(K / F) \rightarrow \operatorname{GL}_{n}\left(\overline{\mathbb{Q}}_{p}\right)$ be an irreducible, self-dual, orthogonal representation. Further assume that

(i) $E_{1}[p](K)=0$.

(ii) $S_{p}\left(E_{1} / K_{\mathrm{cyc}}\right)[p]$ is finite.

Then

$$
\frac{W\left(E_{1} / F, \sigma\right)}{W\left(E_{2} / F, \sigma\right)}=(-1)^{s_{p}\left(E_{1} / F, \sigma\right)-s_{p}\left(E_{2} / F, \sigma\right)} .
$$

In particular, the p-parity conjecture stated in (2) holds for the twist of $E_{1} / F$ by $\sigma$ if and only if it holds for the twist of $E_{2} / F$ by $\sigma$.

Using Theorem 3.5, we give new examples of $E, F, K$ and $\sigma$ (see Example $3.9 \&$ Example 3.11) such that the $p$-parity conjecture in (2) holds for $(E, F, \sigma)$, under some mild condition related to certain $\mu$-invariants.

In Theorem 3.5, it is assumed that $\sigma$ is orthogonal. However, as we point out in Remark 3.7, when $\sigma$ is symplectic, the results stated in Theorem 3.5 are known to hold trivially, assuming the non-degeneracy of the $p$-adic height pairing.

In the reverse situation, where $E$ is fixed and $\rho_{1}, \rho_{2}$ are two self-dual Artin representation of $\Delta$ such that $\tilde{\rho}_{1} \cong \tilde{\rho}_{2}$ (see $\S 1$ ), the variation of the $p$-parity conjecture has already been discussed by Greenberg (see Remark 3.8). 
To prove Theorem 3.5, we study the parity of $s_{p}\left(E_{1} / F, \sigma\right)-s_{p}\left(E_{2} / F, \sigma\right)$ and $\frac{W\left(E_{1} / F, \sigma\right)}{W\left(E_{2} / F, \sigma\right)}$ separately. It is well known (Prop. 1.2) that $s_{p}\left(E_{i} / F, \sigma\right)$ and the multiplicity of $\sigma, \lambda_{E_{i}}(\sigma)$ in $S_{p}\left(E_{i}\left[p^{\infty}\right] \otimes \sigma / F_{\text {cyc }}\right)^{\vee}$ have the same parity. Keeping this in mind, we apply Iwasawa theoretic methods to compare the parity of $\lambda_{E_{1}}(\sigma)$ with $\lambda_{E_{2}}(\sigma)$ for the congruent elliptic curves. From this, it reduces to the comparison of the parity of local Iwasawa invariants $\delta_{E_{i}, v}(\sigma)$, for $v \in \Sigma_{0}$; a finite set of primes of $F$, away from $p$. But this Iwasawa theoretic set up works, only when we assume some conditions, which are stated in Hypothesis $(\mathrm{H})$ in $\S 1$. On the other hand, the global root number is given by product of all the local root numbers. Using this, we compare the local root numbers $W\left(E_{i} / F_{v}, \sigma_{v}\right)$ at each prime $v$ and then go on to show that the ratio of the global root numbers of the Artin twists are also given by the parity of precisely the same local Iwasawa invariants, away from $p$; thereby proving Theorem 3.5.

In fact, the comparison of the parity $s_{p}\left(E_{1} / F, \sigma\right)$ with $s_{p}\left(E_{2} / F, \sigma\right)$ is established in Theorem 2.18 and the root numbers of the Artin twists were compared in Theorem 3.4. We now state Theorem 2.18, which we think is also independently interesting. Let $\Sigma$ be a finite set of primes of $F$ containing the primes above $p$, the infinite primes, the primes of bad reduction of $E_{1}$ and $E_{2}$ and primes which ramify in $K / F$ and $\Sigma_{0}$ is certain subset of $\Sigma$ away from $p$, defined in (25). We denote by $S_{i}, N_{i}, W, X, Y_{3}, Z_{3}$ certain subsets of $\Sigma_{0}$, defined in 2.17 .

Theorem 0.2 (Theorem 2.18). Let $p$ be an odd prime and $k$ be a number field such that all primes of $k$ lying above $p$ are ramified in $k\left(\mu_{p}\right) / k$. Let $E_{1}, E_{2}$ be two elliptic curves over $k$ with $E_{1}[p] \cong E_{2}[p]$ as $G_{k}$-modules. Let $\sigma$ be an absolutely irreducible self-dual orthogonal representation of $\Delta$. Assume the hypothesis $\mathbf{( H )}$. Then

$$
\begin{aligned}
& s_{p}\left(E_{1}, \sigma\right)+\sum_{v \in S_{1}}\left\langle\sigma_{v}, 1_{v}\right\rangle+\sum_{v \in N_{1}}\left\langle\sigma_{v}, \varkappa_{v}\right\rangle+\sum_{v \in W}\left(\left\langle\sigma_{v}, \vartheta_{v}\right\rangle+\left\langle\sigma_{v}, \omega_{v} \vartheta_{v}\right\rangle\right)+\sum_{v \in Z_{3}}\left(\left\langle\sigma_{v}, \omega_{v} \vartheta_{v}\right\rangle+\left\langle\sigma_{v}, \vartheta_{v} \theta_{3, v}\right\rangle\right) \\
& \left.\equiv s_{p}\left(E_{2}, \sigma\right)+\sum_{v \in S_{2}}\left\langle\sigma_{v}, 1_{v}\right\rangle+\sum_{v \in N_{2}}\left\langle\sigma_{v}, \varkappa_{v}\right\rangle+\sum_{v \in X}\left\langle\sigma_{v}, \vartheta_{v}\right\rangle+\sum_{v \in Y_{3}}\left(\left\langle\sigma_{v}, 1_{v}\right\rangle+\left\langle\sigma_{v}, \varkappa_{v}\right\rangle+\left\langle\sigma_{v}, \theta_{3, v}\right\rangle\right) \quad \text { (mod } 2\right) .
\end{aligned}
$$

(Here $\langle\beta, \alpha\rangle$ denotes the multiplicity of $\alpha$ in $\beta$, see $\S 1$ for the definitions of the representations involved.)

We would like to mention that our proof is more involved when the prime $p=3$. In addition, we also need delicate arguments to compare the local Iwasawa invariants $\delta_{E_{i}, v}(\sigma)$ and local root number of Artin twists at primes $v$ of additive reductions, away from $p$. Moreover, the study of $\delta_{E_{1}, v}(\sigma)-\delta_{E_{2}, v}(\sigma)$ and $W\left(E_{1} / F_{v}, \sigma_{v}\right) / W\left(E_{2} / F_{v}, \sigma_{v}\right)$ at primes $v \nmid p$ of $F$ such that $v \mid 2$ or $v \mid 3$ needs careful consideration. We have covered all such possibilities without any exception.

Mazur-Rubin have developed arithmetic theory of local constants which has been used extensively in Nekovář's study of the p-parity conjecture. Nekovář's setting applies when we make an additional hypothesis that the Galois isomorphism between $E_{1}[p]$ and $E_{2}[p]$ is symplectic. In this symplectic isomorphism setting, Nekovár [Ne15] expresses the ratio of the local root numbers of two congruent mod- $p$ curves, at a prime $v \nmid p$, in terms of arithmetic local constant at that prime. Moreover, using Mazur-Rubin's results, it is possible to express the Selmer corank in terms of arithmetic local constants at various primes, including primes dividing $p$ (see (41)). This naturally suggests that the parity of our local Iwasawa invariants $\delta_{E_{i}, v}(\sigma), v \nmid p$ are related to this arithmetic local constant. We establish this relation by directly comparing the difference of the local Iwasawa invariants at a prime away from $p$ with 
the arithmetic local constant at that prime (Proposition 4.3). We can think of this phenomenon as an Iwasawa theoretic interpretation of the arithmetic local constants.

However, as mentioned in Remark 4.1, the situation at $v=p$ is more complicated (see [Ne15] and his subsequent works) and even in the symplectic isomorphism setting, we believe Theorem 3.5 does not follow only from the theory of arithmetic local constants.

Our results are a generalization of results obtained in [She16] and [AAS17]. In particular, when $\sigma$ is the trivial representation, [AAS17, Theorem 5.5] follows from our Theorem 3.5 and [She16, Theorem 1.1] follows from our Theorem 2.18. We stress that the results in [She16] and [AAS17] only concerns the $p$-parity conjecture for the trivial twist and this generalization to a non-trivial twist in this article is not straightforward. The $p$-parity conjecture, for a non-trivial twist is much less understood, compared to the trivial twist case (cf. [DD09, Theorem 1.3]) and we undertake this study in this article. Also, we give a direct comparison and description of Mazur-Rubin-Nekovarřs's arithmetic local constants in terms of (a priori different) explicit local Iwasawa invariants (Proposition 4.3); this was not considered earlier. Further, in this article, we give new examples of $E, F, K, \sigma$ such that the $p$-parity conjecture for the twist of $E / F$ by $\sigma$ holds, under some mild assumption related to certain $\mu$-invariants (Examples $3.9 \& 3.11$ ).

The summary and structure of this article is as follows: The basic algebraic set up is discussed in section 1 and we collect all the relevant known results related to $s_{p}(E / F, \sigma)$ in this section, many of which are due to Greenberg. In subsection 2.1, we consider imprimitive Selmer groups and prove that the multiplicity of $\sigma$ in the imprimitive $\Sigma_{0}$ Selmer group, with our suitably chosen $\Sigma_{0}$, coincides for the congruent mod- $p$ elliptic curves. Note that, here we make an improvement on methods of [GV00], by choosing a $\Sigma_{0}$ which is smaller than the corresponding set of [GV00]. This reduces the study of $s_{p}\left(E_{1} / F, \sigma\right)-s_{p}\left(E_{2} / F, \sigma\right)(\bmod 2)$ to the study of local invariants at $\delta_{E_{1}, v}(\sigma)-\delta_{E_{2}, v}(\sigma)$ for each $v \in \Sigma_{0}$, which we do in subsection 2.2. Combining these results, we prove Theorem 2.18 in subsection 2.3, which completes the comparison of $s_{p}\left(E_{i} / F, \sigma\right)$. Subsection 3.1 contains basic known results on root numbers, many of them are due to Rohrlich. We compare the root numbers $\frac{W\left(E_{1} / F, \sigma\right)}{W\left(E_{2} / F, \sigma\right)}$ in subsection 3.2 and express it in terms of local Iwasawa invariants $\delta_{E_{i}, v}(\sigma)$, where $v \in \Sigma_{0}$. Using this, we prove our main result, Theorem 3.5 in subsection 3.3. Then we discuss numerical examples of $p$-parity conjecture for an Artin twist of an elliptic curve, as applications of our result, in subsection 3.4. Finally, in section 4, we directly compare local Iwasawa invariants at a prime away from $p$ with Mazur-Rubin and Nekovářss arithmetic local constants and show our methods are consistent with the theory of arithmetic local constants.

Notation: For a number field or an $\ell$-adic field $L, L_{\text {cyc }}$ will be the cyclotomic $\mathbb{Z}_{p}$-extension of $L$ with $\Gamma:=\operatorname{Gal}\left(L_{\text {cyc }} / L\right) \cong \mathbb{Z}_{p}$. Recall, the Iwasawa algebra of $\Gamma$, denoted by $\Lambda$, is defined as $\Lambda=\mathbb{Z}_{p}[[\Gamma]]:=$ ${\underset{\varliminf}{n}}_{\lim _{p}} \mathbb{Z}_{p}\left[\frac{\Gamma}{\Gamma^{n}}\right]$. For a discrete $\Lambda$ module $M$, we denote the Pontryagin dual $\operatorname{Hom}_{\text {cont }}\left(M, \mathbb{Q}_{p} / \mathbb{Z}_{p}\right)$ by $M^{\vee}$. For any separable field $L, G_{L}$ will denote the Galois group $\operatorname{Gal}(\bar{L} / L)$ and $I_{L}$ denotes the inertia subgroup of $G_{L}$. For an algebraic extension $L$ of $\mathbb{Q}$, the completion of $L$ at a prime $v$ of $L$ is denoted by $L_{v}$.

\section{The ALgEBRAIC SET UP}

Let $p$ be an odd prime and $\mu_{p}$ denotes the group of $p$-th roots of unity. Let $k$ be a number field such that all primes of $k$ lying above $p$ are ramified in $k\left(\mu_{p}\right) / k$. Now $F$ be any number field containing $k$. 
Consider a finite Galois extension $K / F$ such that $K \cap F_{\text {cyc }}=F$, where $F_{\text {cyc }}$ is the cyclotomic $\mathbb{Z}_{p}$-extension of $F$. We set $\Delta:=\operatorname{Gal}(K / F)$.

Let $E$ be an elliptic curve over $k$ with good, ordinary reduction at all primes of $k$ lying above $p$. Let $N_{E}^{F}$ and $\bar{N}_{E}^{F}$ respectively denote the conductor of $E$ and the prime-to- $p$ part of the conductor of the $G_{F}$ module $E[p]$. Here the conductor of $E[p]$ is defined following Serre and can be found in [Li89, $\S 1$, Page 135]. Let $T_{p} E$ denote the Tate module of $E$ and $\rho_{E}: G_{k} \rightarrow \operatorname{Aut}\left(T_{p} E\right) \cong \mathrm{GL}_{2}\left(\mathbb{Z}_{p}\right)$ be the Galois representation associated to $E$. Let $\Sigma$ be a finite set of places of $F$ containing the primes of bad reduction of $E$, the primes above $p$, the infinite primes and primes that ramify in $K / F$. We write $F_{\Sigma}$ for the maximal algebraic extension of $F$ unramified outside $\Sigma$.

Given an irreducible Artin representation $\sigma: \Delta \longrightarrow \mathrm{GL}_{n}\left(\overline{\mathbb{Q}}_{p}\right)$, we can view $\sigma$ as an absolutely irreducible representation $\sigma: \Delta \longrightarrow \mathrm{GL}_{n}(\mathcal{F})$, where $\mathcal{F}$ is some finite extension of $\mathbb{Q}_{p}$ with ring of integer $O$, residue field $\mathfrak{f}$ and uniformizer $\pi$. Let $L_{\sigma}$ be a $\Delta$-invariant $O$-lattice in the underlying $\mathcal{F}$ representation space $W_{\sigma}$ for $\sigma$ and set $\operatorname{dim}(\sigma)=\operatorname{dim}_{\mathcal{F}} W_{\sigma}$. We will denote the $G_{F}$ module $E\left[p^{\infty}\right] \otimes_{\mathbb{Z}_{p}} L_{\sigma}$ by $E\left[p^{\infty}\right] \otimes \sigma$. Reducing modulo $\pi$, we obtain a representation $\widetilde{\sigma}$ over $\mathfrak{f}$ and let $U_{\widetilde{\sigma}}$ denote the $\mathfrak{f}$-representation space for $\widetilde{\sigma}$. The $G_{F}$ module $E[p] \otimes_{\mathbb{F}_{p}} U_{\widetilde{\sigma}}$ is denoted by $E[p] \otimes \widetilde{\sigma}$. We have $\left(E\left[p^{\infty}\right] \otimes \sigma\right)[\pi] \cong E[p] \otimes \widetilde{\sigma}$.

Let $v, u, w, \eta$ respectively denote primes in $F, K, F_{\text {cyc }}, K_{\text {cyc }}$ such that $\eta|u| v$ and $\eta|w| v$. Let $\widetilde{E}_{v}$ denote the reduction of $E$ at a prime $v \mid p$ of $F$. As $E$ is good, ordinary at all primes $v$ above $p$, $\widetilde{E}_{v}\left[p^{\infty}\right]:=\bigcup_{n} \widetilde{E}_{v}\left[p^{n}\right]$ is an unramified $G_{F_{v}}$-module for every $v \mid p$. Set

$$
\begin{gathered}
\mathcal{H}_{v}\left(F_{\mathrm{cyc}}, E\left[p^{\infty}\right] \otimes \sigma\right):= \begin{cases}\prod_{w \mid v} \mathrm{H}^{1}\left(F_{\mathrm{cyc}, w}, E\left[p^{\infty}\right] \otimes \sigma\right) & \text { if } v \nmid p \\
\prod_{w \mid v} \mathrm{H}^{1}\left(F_{\mathrm{cyc}, w}, \widetilde{E}_{v}\left[p^{\infty}\right] \otimes \sigma\right) & \text { if } v \mid p .\end{cases} \\
\mathcal{H}_{v}\left(F_{\mathrm{cyc}}, E[p] \otimes \widetilde{\sigma}\right):= \begin{cases}\prod_{w \mid v} \mathrm{H}^{1}\left(F_{\mathrm{cyc}, w}, E[p] \otimes \widetilde{\sigma}\right) & \text { if } v \nmid p \\
\prod_{w \mid v} \mathrm{H}^{1}\left(F_{\mathrm{cyc}, w}, \widetilde{E}_{v}[p] \otimes \widetilde{\sigma}\right) & \text { if } v \mid p .\end{cases}
\end{gathered}
$$

and

$$
\mathcal{H}_{u}\left(K_{\mathrm{cyc}}, E\right):= \begin{cases}\prod_{\eta \mid u} \mathrm{H}^{1}\left(K_{\mathrm{cyc}, \eta}, E\left[p^{\infty}\right]\right) & \text { if } u \mid v, u \nmid p, v \in \Sigma . \\ \prod_{\eta \mid u} \mathrm{H}^{1}\left(K_{\mathrm{cyc}, \eta}, \widetilde{E}_{v}\left[p^{\infty}\right]\right) & \text { if } u \mid p .\end{cases}
$$

For any subset $\Sigma^{\prime} \subset \Sigma \backslash\{v$ prime in $F: v \mid p \infty\}$, we define various imprimitive $\Sigma^{\prime}$-Selmer groups as follows:

$$
\begin{gathered}
0 \longrightarrow S_{p}^{\Sigma^{\prime}}\left(E\left[p^{\infty}\right] \otimes \sigma / F_{\mathrm{cyc}}\right) \longrightarrow \mathrm{H}^{1}\left(F_{\Sigma} / F_{\mathrm{cyc}}, E\left[p^{\infty}\right] \otimes \sigma\right) \stackrel{\phi}{\longrightarrow} \prod_{v \in \Sigma \backslash \Sigma^{\prime}} \mathcal{H}_{v}\left(F_{\mathrm{cyc}}, E\left[p^{\infty}\right] \otimes \sigma\right) . \\
0 \longrightarrow S_{p}^{\Sigma^{\prime}}\left(E[p] \otimes \widetilde{\sigma} / F_{\mathrm{cyc}}\right) \longrightarrow \mathrm{H}^{1}\left(F_{\Sigma} / F_{\mathrm{cyc}}, E[p] \otimes \widetilde{\sigma}\right) \longrightarrow \prod_{v \in \Sigma \backslash \Sigma^{\prime}} \mathcal{H}_{v}\left(F_{\mathrm{cyc}}, E[p] \otimes \widetilde{\sigma}\right) . \\
0 \longrightarrow S_{p}^{\Sigma^{\prime}}\left(E / K_{\mathrm{cyc}}\right) \longrightarrow \mathrm{H}^{1}\left(F_{\Sigma} / K_{\mathrm{cyc}}, E\left[p^{\infty}\right]\right) \longrightarrow \prod_{u \mid v, v \in \Sigma \backslash \Sigma^{\prime}} \mathcal{H}_{u}\left(K_{\mathrm{cyc}}, E\right),
\end{gathered}
$$

We also recall that by our choice of $\Sigma$, we have

$$
0 \longrightarrow S_{p}\left(E / K_{\mathrm{cyc}}\right) \longrightarrow \mathrm{H}^{1}\left(F_{\Sigma} / K_{\mathrm{cyc}}, E\left[p^{\infty}\right]\right) \longrightarrow \prod_{u \mid v, v \in \Sigma} \mathcal{H}_{u}\left(K_{\mathrm{cyc}}, E\right)
$$


Now assume that $E$ has good, ordinary reduction at all primes $v$ of $F$ dividing $p$ and also assume that $S_{p}\left(E / K_{\text {cyc }}\right)[p]$ is finite. Then $S_{p}\left(E / K_{\text {cyc }}\right)^{\vee}$ is a finitely generated $\mathbb{Z}_{p}$-module. For any irreducible representation $\sigma$ of $\Delta$ over $\mathcal{F}$, we define $\lambda_{E}(\sigma)$ (respectively $\lambda_{E}^{\Sigma^{\prime}}(\sigma)$ ) to be the multiplicity of $\sigma$ in the $\mathcal{F}$ representation space $S_{p}\left(E / K_{\text {cyc }}\right)^{\vee} \otimes_{\mathbb{Z}_{p}} \mathcal{F}$ (respectively $S_{p}^{\Sigma^{\prime}}\left(E / K_{\text {cyc }}\right)^{\vee} \otimes_{\mathbb{Z}_{p}} \mathcal{F}$.

Then under the hypotheses that $E$ is good, ordinary at primes dividing $p$ with $S_{p}\left(E / K_{\text {cyc }}\right)[p]$ finite, it is shown [Gre11, Proposition 4.3.1] that

$$
\lambda_{E}^{\Sigma^{\prime}}(\sigma)=\operatorname{rank}_{O} S_{p}^{\Sigma^{\prime}}\left(E\left[p^{\infty}\right] \otimes \sigma / F_{\mathrm{cyc}}\right)^{\vee} .
$$

Moreover we have the following result of Greenberg:

Lemma 1.1. [Gre11, Prop. 4.3.3] Let $E$ be good, ordinary at primes of $F$ dividing $p$. Assume that either $E[p](K)=0$ or $\Sigma^{\prime}$ is non-empty. Also assume that $S_{p}\left(E / K_{\text {cyc }}\right)[p]$ is finite. Then $S_{p}^{\Sigma^{\prime}}\left(E\left[p^{\infty}\right] \otimes \sigma / F_{\text {cyc }}\right)$ is $\pi$-divisible. In particular, the maximal pseudo-null $\Lambda$-submodule of $S_{p}^{\Sigma^{\prime}}\left(E\left[p^{\infty}\right] \otimes \sigma / F_{\text {cyc }}\right)^{\vee}$ is 0 .

By Lemma 1.1, it follows from (11) that

$$
\lambda_{E}^{\Sigma^{\prime}}(\sigma)=\operatorname{rank}_{f} \frac{S_{p}^{\Sigma^{\prime}}\left(E\left[p^{\infty}\right] \otimes \sigma / F_{\mathrm{cyc}}\right)^{\vee}}{\pi} .
$$

Recall, $S_{p}\left(E / K_{\text {cyc }}\right)[p]$ is finite implies that $S_{p}\left(E / K_{\text {cyc }}\right)$ is a cotorsion $\Lambda$-module. Then it is well known that the global to local maps in (10) and (6) are surjective and hence we have (see for example [Gre11, (1.3.a)])

$$
S_{p}^{\Sigma^{\prime}}\left(E / K_{\mathrm{cyc}}\right) / S_{p}\left(E / K_{\mathrm{cyc}}\right) \cong \prod_{u \mid v, v \in \Sigma^{\prime}} \mathcal{H}_{u}\left(K_{\mathrm{cyc}}, E\right)
$$

Let $\delta_{E}^{\Sigma^{\prime}}(\sigma)$ denote the multiplicity of $\sigma$ in the $\mathcal{F}$ representation space $\left(\prod_{v \in \Sigma^{\prime}} \mathcal{H}_{v}\left(K_{\text {cyc }}, E\right)\right)^{\vee} \otimes_{\mathbb{Z}_{p}}$ $\mathcal{F}$. For a prime $w \mid v$ of $F_{\text {cyc }}$, we also consider the multiplicity of $\sigma$ in the $\mathcal{F}$ representation space $\left(\prod_{\eta \mid w} \mathrm{H}^{1}\left(K_{\mathrm{cyc}, \eta}, E\left[p^{\infty}\right]\right)\right)^{\vee} \otimes_{\mathbb{Z}_{p}} \mathcal{F}$. This last multiplicity of $\sigma$ in $\left(\prod_{\eta \mid w} \mathrm{H}^{1}\left(K_{\mathrm{cyc}, \eta}, E\left[p^{\infty}\right]\right)\right)^{\vee} \otimes_{\mathbb{Z}_{p}} \mathcal{F}$ is the same for two different $w$ and $w^{\prime}$ in $F_{\text {cyc }}$ lying over a prime $v$ in $F$ [Gre11, (5.0.b)]; hence it is denoted by $\delta_{E, v}(\sigma)$. Let $s_{v}$ denote the number of primes of $F_{\text {cyc }}$ lying above $v$. Then from (13), we obtain:

$$
\lambda_{E}^{\Sigma^{\prime}}(\sigma)=\lambda_{E}(\sigma)+\delta_{E}^{\Sigma^{\prime}}(\sigma)=\lambda_{E}(\sigma)+\sum_{v \in \Sigma^{\prime}} s_{v} \delta_{E, v}(\sigma) .
$$

Recall an absolutely irreducible, self-dual representation $\sigma$ of $\Delta$ over $\mathcal{F}$ is said to be orthogonal (respectively symplectic) if the non-degenerate $\Delta$-invariant pairing $B: W_{\sigma} \times W_{\sigma} \rightarrow \mathcal{F}$ is symmetric (respectively skew-symmetric), where $W_{\sigma}$ denotes the underlying representation space for $\sigma$. The following relation between $\lambda_{E}(\sigma)$ and $s_{p}(E, \sigma)$ was observed by Greenberg.

Proposition 1.2. [Gre10, Proposition 4.1] Let $E$ be an elliptic curve over $K$. If $S_{p}\left(E / K_{\mathrm{cyc}}\right)$ is $\Lambda$ cotorsion, then for an irreducible orthogonal representation $\sigma$ of $\Delta$, we have

$$
\lambda_{E}(\sigma) \equiv s_{p}(E, \sigma) \quad(\bmod 2) .
$$

Note that $s_{v}$ in (14) is odd, as $p$ is odd. Then using (15) and (14), we deduce the following corollary: 
Corollary 1.3. Let $E$ be good, ordinary at primes of $F$ dividing $p$. Assume that either $E[p]$ is an irreducible $G_{K}$ module or that $\Sigma^{\prime}$ is non-empty. We further assume that $S_{p}\left(E / K_{\mathrm{cyc}}\right)[p]$ is finite. Then for an irreducible orthogonal representation $\sigma$ of $\Delta$, we get

$$
s_{p}(E, \sigma) \equiv \lambda_{E}^{\Sigma^{\prime}}(\sigma)+\sum_{v \in \Sigma^{\prime}} \delta_{E, v}(\sigma) \quad(\bmod 2) .
$$

Definition 1.4. Given a finite group $G$, we consider two finite dimensional representations $\alpha$ and $\beta$ over a field $L$ with $\alpha$ absolutely irreducible. Let $V_{\alpha}$ and $V_{\beta}$ be the corresponding representation spaces. Then define $\langle\beta, \alpha\rangle=\langle\beta, \alpha\rangle_{G}:=\operatorname{dim}_{L} \operatorname{Hom}\left(V_{\alpha}, V_{\beta}\right)$. In other words, $\langle\beta, \alpha\rangle$ is the largest non-negative integer $m$ such that $V_{\beta}$ contains a $G$-invariant subspace isomorphic to $V_{\alpha}^{m}$. Note that if $G$ is a quotient of $\mathcal{G}$, then $\langle\beta, \alpha\rangle_{G}=\langle\beta, \alpha\rangle_{\mathcal{G}}$.

Determination of $\delta_{E, v}(\sigma)$ : As we have noted earlier, given a prime $v$ in $F, \delta_{E_{i}, v}(\sigma)$ does not depend on the choice of $w$ in $F_{\text {cyc }}$ or $\eta$ in $K_{\text {cyc }}$. Hence by abuse of notation, when there is no cause of confusion, we will simply denote $F_{\mathrm{cyc}, w}, K_{\mathrm{cyc}, \eta}$ and $\Delta_{\eta}=\operatorname{Gal}\left(K_{\mathrm{cyc}, \eta} / F_{\mathrm{cyc}, w}\right)$ respectively by $F_{\mathrm{cyc}, v}, K_{\mathrm{cyc}, v}$ and $\Delta_{v}$. Recall $\sigma_{v}:=\left.\sigma\right|_{\Delta_{v}}$ is the restriction of $\sigma$ to the decomposition subgroup of $\Delta$ at $v$. Let $\operatorname{Irr}_{\mathcal{F}}\left(\Delta_{v}\right)$ be the set of all $\mathcal{F}$ irreducible representations of $\Delta_{v}$. By going to a finite extension of $\mathcal{F}$ if necessary, we may assume each $\chi \in \operatorname{Irr}_{\mathcal{F}}\left(\Delta_{v}\right)$ is absolutely irreducible. We view $\rho_{E, v}:=\left.\rho_{E}\right|_{G_{F_{\mathrm{cyc},},}}$ as the representation $\rho_{E, v}: G_{F_{\mathrm{cyc}, v}} \rightarrow V_{p}(E) \otimes_{\mathbb{Q}_{p}} \mathcal{F}$. Then we have the following expression for $\delta_{E, v}(\sigma)$ ([Gre11, (5.1.a), §5.2]).

$$
\delta_{E, v}(\sigma)=\sum_{\chi \in \operatorname{Irr}_{\mathcal{F}}\left(\Delta_{v}\right)}\left\langle\sigma_{v}, \chi\right\rangle\left\langle\rho_{E, v}, \chi\right\rangle .
$$

Corollary 1.5. Let $E$ be as in the setting of Corollary 1.3. Then for an irreducible orthogonal representation $\sigma$ of $\Delta$, we get

$$
s_{p}(E, \sigma) \equiv \lambda_{E}^{\Sigma^{\prime}}(\sigma)+\sum_{v \in \Sigma^{\prime}} \sum_{\chi \in \operatorname{Irr} \mathcal{F}\left(\Delta_{v}\right)}\left\langle\sigma_{v}, \chi\right\rangle\left\langle\rho_{E, v}, \chi\right\rangle \quad(\bmod 2) .
$$

Remark 1.6. Assume that we have two elliptic curves $E_{1}$ and $E_{2}$ which are congruent mod $p$ and they fit into the setting of Corollary 1.5. Then to compare the parity of $s_{p}\left(E_{1}, \sigma\right)$ with $s_{p}\left(E_{2}, \sigma\right)$, by the same corollary, it reduces to compare (i) the Iwasawa invariants $\lambda_{E_{1}}^{\Sigma^{\prime}}(\sigma)$ with $\lambda_{E_{2}}^{\Sigma^{\prime}}(\sigma)$ for a suitably chosen $\Sigma^{\prime}$ and then (ii) compare the parity of $\left\langle\sigma_{v}, \chi\right\rangle\left\langle\rho_{E_{1}, v}, \chi\right\rangle$ with $\left\langle\sigma_{v}, \chi\right\rangle\left\langle\rho_{E_{2}, v}, \chi\right\rangle$ for each $v$ in that particularly chosen set $\Sigma^{\prime}$, with $\chi$ as above. We will do this in the next section.

We now study $\left\langle\rho_{E, v}, \chi\right\rangle$, according to the reduction type of $E$ at $v$. Set $\omega_{v}:=\left.\omega_{p}\right|_{G_{F_{\mathrm{cyc},}}}$, where $\omega_{p}: G_{\mathbb{Q}} \rightarrow \mathbb{Z}_{p}^{\times}$is the $p$-adic cyclotomic character. Then $\omega_{v}$ is a power of the Teichmüller character and the order of $\omega_{v}$ divides $p-1$.

If $E$ has good reduction at $v$, then the action of $G_{F_{\mathrm{cyc}, v}}$ on $V_{p}(E)$ is unramified and after extending $\mathcal{F}$ if necessary, the representation space $V_{p}(E) \otimes_{\mathbb{Q}_{p}} \mathcal{F}$ is the direct sum of two one-dimensional subspaces on which $G_{F_{\text {cyc }, v}}$ acts by unramified characters $\varphi_{E, v}, \psi_{E, v}$ (say) of orders dividing $p^{2}-1$. By Weil pairing, $\varphi_{E, v} \psi_{E, v}=\omega_{v}$.

$$
\left\langle\rho_{E, v}, \chi\right\rangle= \begin{cases}2 & \text { if } \chi=\varphi_{E, v}=\psi_{E, v} \\ 1 & \text { if } \chi \in\left\{\varphi_{E, v}, \psi_{E, v}\right\} \text { and } \varphi_{E, v} \neq \psi_{E, v} \\ 0 & \text { otherwise }\end{cases}
$$


Let $E$ be multiplicative at $v$ and $\varkappa_{v}$ is the unique unramified quadratic character of $G_{F_{\mathrm{cyc}, v}}$. By [DDT94, Prop. 2.12]

$$
\rho_{E, v} \sim\left(\begin{array}{cc}
\omega_{v} & * \\
0 & 1
\end{array}\right) \text {, if } E \text { splits at } v \text { and } \rho_{E, v} \sim\left(\begin{array}{cc}
\omega_{v} & * \\
0 & 1
\end{array}\right) \otimes \varkappa_{v} \text {, if } E \text { is non-split at } v .
$$

If $E$ is split at $v,\left\langle\rho_{E, v}, \chi\right\rangle=\left\{\begin{array}{ll}1 & \text { if } \chi=\omega_{v} \\ 0 & \text { otherwise. }\end{array} \quad\right.$ If $E$ is non-split at $v,\left\langle\rho_{E, v}, \chi\right\rangle= \begin{cases}1 & \text { if } \chi=\omega_{v} \varkappa_{v} \\ 0 & \text { otherwise. }\end{cases}$

If $E$ has additive but potentially multiplicative reduction at $v$, then $E$ is split multiplicative over a ramified quadratic extension of $F_{\text {cyc }, v}$. Also $V_{p} E$ has a unique one dimensional $\rho_{E, v}$ invariant subspace on which $\rho_{E, v}$ acts via $\omega_{v} \vartheta_{E, v}$ (say), where $\vartheta_{E, v}$ is a ramified quadratic character of $G_{F_{\mathrm{cyc}, v}}$ associated with the extension $F_{v}\left(\sqrt{-c_{6}}\right) / F_{v}$, where $c_{6}$ is coming from the Weierstrass equation of $E$ [Sil94, Page 442-444]. Then

$$
\begin{gathered}
\rho_{E, v} \sim\left(\begin{array}{cc}
\omega_{v} & * \\
0 & 1
\end{array}\right) \otimes \vartheta_{E, v} . \\
\text { In this case, } \quad\left\langle\rho_{E, v}, \chi\right\rangle= \begin{cases}1 & \text { if } \chi=\omega_{v} \vartheta_{E, v} \\
0 & \text { otherwise }\end{cases}
\end{gathered}
$$

If $E$ has additive but potentially good reduction at $v$, then $\rho_{E}\left(G_{F_{\mathrm{cyc}, v}}\right)$ is finite as we are over cyclotomic extension [Gre11, Page 73]. Moreover, for $p \geq 5, p \nmid \# \rho_{E}\left(G_{F_{\mathrm{cyc}, v}}\right)$. If $\rho_{E}\left(G_{F_{\mathrm{cyc}, v}}\right)$ is abelian, then the $G_{F_{\mathrm{cyc}, v}}$ representation $V_{p}(E) \otimes \mathcal{F}$ is a direct sum of two characters, say $\varepsilon_{E, v}, \varepsilon_{E, v}^{-1} \omega_{v}$. If $\rho_{E}\left(G_{F_{\mathrm{cyc}, v}}\right)$ is non-abelian, then $\rho_{E, v}$ is absolutely irreducible. Thus

$$
\left\langle\rho_{E, v}, \chi\right\rangle= \begin{cases}2 & \text { if } \chi=\varepsilon_{E, v}=\varepsilon_{E, v}^{-1} \omega_{v} \text { and } \rho_{E}\left(G_{F_{\mathrm{cyc}, v}}\right) \text { is abelian } \\ 1 & \text { if } \chi \in\left\{\varepsilon_{E, v}, \varepsilon_{E, v}^{-1} \omega_{v}\right\}, \varepsilon_{E, v} \neq \varepsilon_{E, v}^{-1} \omega_{v} \text { and } \rho_{E}\left(G_{F_{\mathrm{cyc}, v}}\right) \text { is abelian } \\ 1 & \text { if } \chi=\rho_{E, v} \text { and } \rho_{E}\left(G_{F_{\mathrm{cyc}, v}}\right) \text { is non-abelian } \\ 0 & \text { otherwise. }\end{cases}
$$

We end this section with a technical lemma to be used later. Set $S^{*}\left(E[p] \otimes \widetilde{\sigma} / F_{\text {cyc }}\right):={\underset{n}{n}}_{\lim _{p}} S_{\Sigma^{\prime}}(E[p] \otimes$ $\left.\tilde{\sigma} / F_{n}\right)$ where $F_{n}$ varies over the cyclotomic tower $F_{\text {cyc }} / F$. The following lemma follows from the proof of [LS18, Lemma 5.5]. As mentioned by [LS18], their proof follows essentially the idea of [HV03, Prop 7.1].

Lemma 1.7. There is an injective $\operatorname{map} S^{*}\left(E[p] \otimes \widetilde{\sigma} / F_{\mathrm{cyc}}\right) \hookrightarrow \operatorname{Hom}_{\mathbb{F}_{p}[[\Gamma]]}\left(S_{p}^{\Sigma^{\prime}}\left(E[p] \otimes \widetilde{\sigma} / F_{\mathrm{cyc}}\right)^{\vee}, \mathbb{F}_{p}[[\Gamma]]\right)$.

\section{Comparison of the PARity of $s_{p}\left(E_{i}, \sigma\right)$}

Now let $E_{1}, E_{2}$ be two elliptic curves over $k$ such that $E_{1}[p] \cong E_{2}[p]$ as $G_{k}$ modules. Recall from introduction, $N_{i}^{F}=N_{E_{i}}^{F}$ denote the conductor of $E_{i}$ over $F$ and $\bar{N}_{i}^{F}=\bar{N}_{E_{i}}^{F}$ denote the prime-to-p part of the conductor of the Galois module $E_{i}[p]$ over $F$ for $i=1,2$. Let $\Sigma=\Sigma\left(E_{1}, E_{2}, K, F\right)$ be a finite set of primes of $F$ containing the primes above $p$, the infinite primes, the primes of bad reduction of both 
$E_{1}$ and $E_{2}$ and primes which ramify in $K / F$. For a prime $v$ of $F$, let $e_{v}(K / F)$ denote the ramification index of $v$ in $K / F$. We now choose and fix a subset $\Sigma_{0}=\Sigma_{0}\left(E_{1}, E_{2}, K, F\right)$ of $\Sigma$ defined as:

$$
\Sigma_{0}=\Sigma_{0}\left(E_{1}, E_{2}, K, F\right):=\left\{v \in \Sigma: v \mid N_{1}^{F} / \bar{N}_{1}^{F} \text { or } v \mid N_{2}^{F} / \bar{N}_{2}^{F}\right\} \cup\left\{v: v \nmid p \infty, \text { and } p \mid e_{v}(K / F)\right\} .
$$

With this definition, we can apply the results of $\S 1$ on imprimitive Selmer groups with $\Sigma^{\prime}=\Sigma_{0}$. Throughout the article, we make the following hypothesis $(\mathbf{H})$.

Hypothesis (H): All of (H1) to (H4) hold simultaneously.

(H1) $E_{1}$ and $E_{2}$ have good reduction at primes of $k$ lying above $p$.

(H2) $E_{1}$ has ordinary reduction at primes of $k$ lying above $p$.

(H3) $E_{1}[p](K)=0$.

(H4) $S_{p}\left(E_{1} / K_{\text {cyc }}\right)[p]$ is finite.

Remark 2.1. It is known (see [She16, Remark 1.2]), the assumptions (H2), (H3) and (H4) hold for $E_{1}$ if and only if the same hold for $E_{2}$. Also (H4) actually implies (H2), by a result of P. Schneider.

As explained in Remark 1.6, to study $s_{p}\left(E_{1}, \sigma\right)-s_{p}\left(E_{2}, \sigma\right)$ (mod 2), we will first compare $\lambda_{E_{1}}^{\Sigma_{0}}(\sigma)$ with $\lambda_{E_{2}}^{\Sigma_{0}}(\sigma)$ and then further calculate $\delta_{E_{1}, v}(\sigma)-\delta_{E_{2}, v}(\sigma)(\bmod 2)$, for each $v \in \Sigma_{0}$.

2.1. Comparison of the parity of the multiplicities $\lambda_{E_{i}}^{\Sigma_{0}}(\sigma)$. The multiplication by $p$ map on $E_{i}$, via Kummer theory, induces a natural map $S_{p}^{\Sigma_{0}}\left(E_{i}[p] \otimes \tilde{\sigma} / F_{\text {cyc }}\right) \longrightarrow S_{p}^{\Sigma_{0}}\left(E_{i}\left[p^{\infty}\right] \otimes \sigma / F_{\text {cyc }}\right)[\pi]$. We compare the parity of $\lambda_{E_{1}}^{\Sigma_{0}}(\sigma)$ with $\lambda_{E_{2}}^{\Sigma_{0}}(\sigma)$ by studying the kernel and the cokernel of this map keeping in mind that $E_{1}[p] \cong E_{2}[p]$ as Galois modules. We begin with the following Lemma:

Lemma 2.2. Let us fix a compatible choice of primes $v, u, w, \eta$ respectively in $F, K, F_{\mathrm{cyc}}, K_{\mathrm{cyc}}$ such that $\eta|u| v$ and $\eta|w| v$. Let $E \in\left\{E_{1}, E_{2}\right\}$. Assume $v \in \Sigma \backslash \Sigma_{0}$ such that $v \nmid p \infty$. Then the natural map $\gamma_{\eta}$, induced from the multiplication by $p$ map on $E$,

$$
\gamma_{\eta}: \mathrm{H}^{1}\left(K_{\mathrm{cyc}, \eta}, E[p]\right) \rightarrow \mathrm{H}^{1}\left(K_{\mathrm{cyc}, \eta}, E\left[p^{\infty}\right]\right)[\pi],
$$

is injective.

Proof: If $E / K$ has additive reduction at $u$, then by the proof of [AAS17, Lemma 2.2], $\mathrm{H}^{1}\left(K_{\mathrm{cyc}, \eta}, E\left[p^{\infty}\right]\right)=$ $\mathrm{H}^{1}\left(K_{\mathrm{cyc}, \eta}, E[p]\right)=0$ and thus $\operatorname{Ker}\left(\gamma_{\eta}\right)$ in $(26)$ is $=0$. On the other hand, if $u$ as above, is prime of good or multiplicative reduction of $E$ over $K$, then we claim that $u \nmid N_{E}^{K} / \bar{N}_{E}^{K}$. Assume the claim at the moment. Then using [EPW06, Lemma 4.1.2], we deduce $E\left[p^{\infty}\right]\left(K_{\mathrm{cyc}, \eta}\right)$ is divisible. By inflationrestriction sequence, we know $\operatorname{Ker}\left(\gamma_{\eta}\right) \cong \mathrm{H}^{0}\left(K_{\mathrm{cyc}, \eta}, E\left[p^{\infty}\right]\right) / \pi$ and hence the $\operatorname{Ker}\left(\gamma_{\eta}\right)$ in (26) is trivial. Thus it reduces to establish the claim that $u \nmid N_{E}^{K} / \bar{N}_{E}^{K}$ to complete the proof of the lemma.

If $E$ is good at $v$ over $F$, then it remains good over at a prime dividing $v$ in any extension of $F$; in particular, $u \nmid N_{E}^{K}$.

Next assume that $E / F$ has multiplicative reduction at $v$; then the same is true for $E / K$ at $u$; in particular $v \| N_{E}^{F}, u|| N_{E}^{K}$ and $\left.\bar{\rho}_{E}\right|_{I_{v}} \sim\left(\begin{array}{ll}1 & * \\ 0 & 1\end{array}\right)$ [DDT94, Proposition 2.12]. Thus, the image of $\left.\bar{\rho}_{E}\right|_{I_{v}}$ has order $p$ or 1, depending on $*$. Recall, by our hypothesis, $v \nmid N_{E}^{F} / \bar{N}_{E}^{F}$ and hence $v \| \bar{N}_{E}^{F}$. If $u \mid N_{E}^{K} / \bar{N}_{E}^{K}$, then we have $u \nmid \bar{N}_{E}^{K}$. Hence, $\left.\bar{\rho}_{E}\right|_{G_{K}}$ is unramified at $u$. So $\# \bar{\rho}_{E}\left(I_{v}\right)=\# \bar{\rho}_{E}\left(I_{v} / I_{u}\right)=1$ as $p \nmid e_{v}(K / F)$. Therefore, $v \nmid \bar{N}_{E}^{F}$ which is a contradiction. 
Now assume that $E / F$ has additive reduction at $v$ i.e. $v^{2} \mid N_{E}^{F}$. Then $v \nmid N_{E}^{F} / \bar{N}_{E}^{F}$ implies that $v^{2} \mid \bar{N}_{E}^{F}$ and so $\left.\bar{\rho}_{E}\right|_{G_{F}}$ is ramified at $v$. If $E / K$ becomes good at $u$, we have $u \nmid N_{E}^{K}$. Thus it reduced to consider the case where $E / K$ is multiplicative at $u$ i.e. $u \| N_{E}^{K}$. If $\left.\bar{\rho}_{E}\right|_{G_{K}}$ is unramified at $u$, then again using $p \nmid e_{v}(K / F)$, we obtain that $\# \bar{\rho}_{E}\left(I_{v}\right)=\# \bar{\rho}_{E}\left(I_{v} / I_{u}\right)=1$, contradicting $\left.\bar{\rho}_{E}\right|_{G_{F}}$ is ramified at $v$. Therefore, $\left.\bar{\rho}_{E}\right|_{G_{K}}$ is ramified at $u$ and so $u \mid \bar{N}_{E}^{K}$. As $u \| N_{E}^{K}, u \nmid N_{E}^{K} / \bar{N}_{E}^{K}$. This completes the proof of the Lemma.

Proposition 2.3. Let $E \in\left\{E_{1}, E_{2}\right\}$ and $v$, in the setting of Lemma 2.2, be a prime in $\Sigma \backslash \Sigma_{0}$ such that $v \nmid p \infty$. Then for any prime $w$ in $F_{\text {cyc }}$ with $w \mid v$, the natural map $\gamma_{w}$ induced by Kummer theory,

$$
\gamma_{w}: \mathrm{H}^{1}\left(F_{\mathrm{cyc}, w}, E[p] \otimes \widetilde{\sigma}\right) \rightarrow \mathrm{H}^{1}\left(F_{\mathrm{cyc}, w}, E\left[p^{\infty}\right] \otimes \sigma\right)[\pi],
$$

is injective.

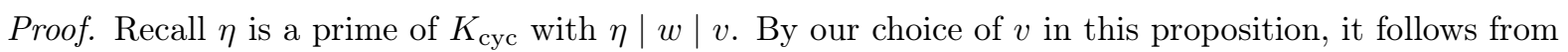
Lemma 2.2 that $\gamma_{\eta}$ in (26) is injective.

Note that as $K \cap F_{\text {cyc }}=F$, we can identify $\Delta \cong \operatorname{Gal}\left(K_{\text {cyc }} / F_{\text {cyc }}\right)$ and the decomposition subgroup $\Delta_{\eta} \cong \operatorname{Gal}\left(K_{\mathrm{cyc}, \eta} / F_{\mathrm{cyc}, w}\right)$. As $v \nmid p$ and by our hypothesis $p \nmid e(K / F)$, we deduce $p \nmid \# \Delta_{\eta}$.

Tensoring equation (26) with $L_{\sigma}$, we obtain that the map $\mathrm{H}^{1}\left(K_{\mathrm{cyc}, \eta}, E[p] \otimes \widetilde{\sigma}\right) \rightarrow \mathrm{H}^{1}\left(K_{\mathrm{cyc}, \eta}, E\left[p^{\infty}\right] \otimes\right.$ $\sigma)[\pi]$ is injective. Now using the inflation-restriction sequence, as $p \nmid \Delta_{\eta}$ it follows that $\gamma_{w}: \mathrm{H}^{1}\left(F_{\text {cyc, } w}, E[p] \otimes\right.$ $\widetilde{\sigma}) \rightarrow \mathrm{H}^{1}\left(F_{\mathrm{cyc}, w}, E\left[p^{\infty}\right] \otimes \sigma\right)[\pi]$ is injective as well. This completes the proof of the proposition.

Now, we can prove the main result of this subsection:

Theorem 2.4. Let $p$ be an odd prime and $k$ be a number field such that all primes of $k$ lying above $p$ are ramified in $k\left(\mu_{p}\right) / k$. Let $E_{1}, E_{2}$ be two elliptic curves over $k$ satisfying the hypothesis (H) and $E_{1}[p] \cong E_{2}[p]$ as $G_{k}$-modules. Then $\lambda_{E_{1}}^{\Sigma_{0}}(\sigma)=\lambda_{E_{2}}^{\Sigma_{0}}(\sigma)$.

Proof. We begin by considering the following commutative diagram induced by multiplication by $\pi$ on $E_{i}\left[p^{\infty}\right] \otimes \sigma$, for $i=1,2$ :

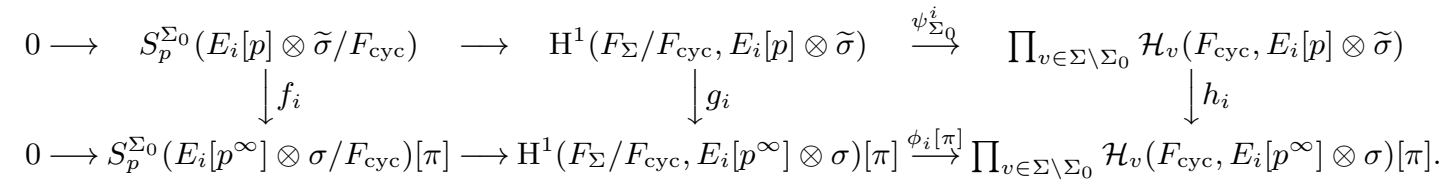

Since $E_{i}\left[p^{\infty}\right] \otimes \sigma$ is a divisible $O$-module, we deduce the middle vertical map $g_{i}$ is surjective. By assumption (H3) and Nakayama's lemma, $E_{i}\left(K_{\mathrm{cyc}}\right)[p]=0$ and hence the map $g_{i}$ is an isomorphism.

Next, we will show that $\psi_{\Sigma_{0}}^{i}$ is surjective. We have the following exact sequence using Poitou-Tate duality:

$$
0 \rightarrow S_{p}^{\Sigma_{0}}\left(E_{i}[p] \otimes \widetilde{\sigma} / F_{\mathrm{cyc}}\right) \longrightarrow \mathrm{H}^{1}\left(F_{\Sigma} / F_{\mathrm{cyc}}, E_{i}[p] \otimes \widetilde{\sigma}\right) \stackrel{\psi_{\Sigma_{0}}^{i}}{\longrightarrow} \prod_{v \in \Sigma \backslash \Sigma_{0}} \mathcal{H}_{v}\left(F_{\mathrm{cyc}}, E_{i}[p] \otimes \widetilde{\sigma}\right) \longrightarrow S^{*}\left(E_{i}[p] \otimes \widetilde{\sigma} / F_{\mathrm{cyc}}\right)^{\vee}
$$

Thus, by Lemma 1.7, to show the surjectivity of $\psi_{\Sigma_{0}}^{i}$, it suffices to show $S_{p}^{\Sigma_{0}}\left(E_{i}[p] \otimes \widetilde{\sigma} / F_{\text {cyc }}\right)$ is finite. Now using Proposition 2.3, $\operatorname{ker}\left(h_{i}\right) \cong \prod_{v \mid p} \prod_{w \mid v} \mathrm{H}^{0}\left(F_{\mathrm{cyc}, w}, \widetilde{E}_{i, v}\left[p^{\infty}\right] \otimes \sigma\right) / \pi$. As the residue field of $K_{\text {cyc }, w}$ for $w \mid p$ is finite, we obtain $\mathrm{H}^{0}\left(F_{\text {cyc }, w}, \widetilde{E}_{i, v}\left[p^{\infty}\right] \otimes \sigma\right)$ is finite and consequently, $\operatorname{ker}\left(h_{i}\right)$ is finite. 
By assumption (H4), $S_{p}\left(E_{i}\left[p^{\infty}\right] / K\right)[p]$ is finite. As $g_{i}$ is an isomorphism and $\operatorname{ker}\left(h_{i}\right)$ is finite, applying snake lemma in diagram (28), it follows that $S_{p}^{\Sigma_{0}}\left(E_{i}[p] \otimes \tilde{\sigma} / F_{\text {cyc }}\right)$ is finite. Thus $\psi_{\Sigma_{0}}^{i}$ is surjective.

Let $v \mid p$ be a prime of $F$. As $p$ is odd and the action of the inertia subgroup of $G_{F_{v}}$ on $\operatorname{ker}\left(E_{i}[p] \rightarrow\right.$ $\left.\bar{E}_{i, v}[p]\right)$ is via the Teichmüller character, by the choice of $k$ we conclude this action is nontrivial. Hence $\bar{E}_{i, v}[p]$ can be characterized as the maximal unramified quotient of $E_{i}[p]$ for $i=1,2$. Now, since $E_{1}[p] \cong E_{2}[p]$ as $G_{k}$-modules, we have $\widetilde{E}_{1, v}[p] \cong \widetilde{E}_{2, v}[p]$ as $G_{F_{v}}$-modules for every prime $v \mid p$ of $F$ (see the preceding paragraph of [She16, Theorem 2.2]). It then follows that $\widetilde{E}_{1, v}[p] \otimes \widetilde{\sigma} \cong \widetilde{E}_{2, v}[p] \otimes \widetilde{\sigma}$ as $G_{F_{v}}$-modules. Then, from the definition of $S_{p}^{\Sigma_{0}}\left(E_{i}[p] \otimes \widetilde{\sigma} / F_{\text {cyc }}\right)$, we obtain that

$$
S_{p}^{\Sigma_{0}}\left(E_{1}[p] \otimes \widetilde{\sigma} / F_{\text {cyc }}\right) \cong S_{p}^{\Sigma_{0}}\left(E_{2}[p] \otimes \widetilde{\sigma} / F_{\text {cyc }}\right) .
$$

In particular, $\operatorname{rank}_{\mathrm{f}} S_{p}^{\Sigma_{0}}\left(E_{1}[p] \otimes \widetilde{\sigma} / F_{\mathrm{cyc}}\right)=\operatorname{rank}_{\mathrm{f}} S_{p}^{\Sigma_{0}}\left(E_{2}[p] \otimes \tilde{\sigma} / F_{\mathrm{cyc}}\right)$. Moreover, notice that

$\operatorname{rank}_{\mathfrak{f}} \mathrm{H}^{0}\left(F_{\mathrm{cyc}, w}, \widetilde{E}_{i, v}\left[p^{\infty}\right] \otimes \sigma\right) / \pi=\operatorname{rank}_{\mathfrak{f}} \mathrm{H}^{0}\left(F_{\mathrm{cyc}, w},\left(\widetilde{E}_{i, v}\left[p^{\infty}\right] \otimes \sigma\right)[\pi]\right)=\operatorname{rank}_{\mathfrak{f}} \mathrm{H}^{0}\left(F_{\mathrm{cyc}, w}, \widetilde{E}_{i, v}[p] \otimes \tilde{\sigma}\right)$

Hence, $\operatorname{rank}_{\mathfrak{f}} \operatorname{ker}\left(h_{1}\right)=\operatorname{rank}_{\mathfrak{f}} \operatorname{ker}\left(h_{2}\right)$.

Now we know $\psi_{\Sigma_{0}}^{i}$ is surjective and $g$ is an isomorphism. Then again applying snake lemma in diagram (28), it is immediate that $\operatorname{rank}_{\mathfrak{f}} S_{p}^{\Sigma_{0}}\left(E_{1}\left[p^{\infty}\right] \otimes \sigma / F_{\text {cyc }}\right)^{\vee} / \pi=\operatorname{rank}_{\mathfrak{f}} S_{p}^{\Sigma_{0}}\left(E_{2}\left[p^{\infty}\right] \otimes \sigma / F_{\text {cyc }}\right)^{\vee} / \pi$. This completes the proof of the theorem.

Remark 2.5. Recall, a prime $v \mid p$ of $F$ is said to be a non-anomalous prime for $E / K$ if $\left|\widetilde{E}_{v}\left(k_{u}\right)\right|$ is not divisible by $p$, for every prime $u \mid v$ of $K$, where $k_{u}$ is the residue field at $u$. As before, assume the hypothesis $(\mathbf{H})$. In addition, assume either (i) $v$ is non-anomalous for $E / K$ or (ii) $\left(\widetilde{E}_{v}\left[p^{\infty}\right] \otimes \sigma\right)^{G_{F_{c y c}, w}}$

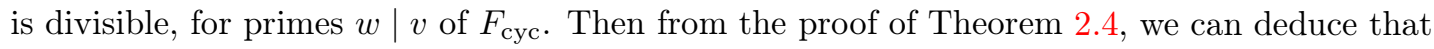

$$
S_{p}^{\Sigma_{0}}\left(E[p] \otimes \widetilde{\sigma} / F_{\text {cyc }}\right) \cong S_{p}^{\Sigma_{0}}\left(E\left[p^{\infty}\right] \otimes \sigma / F_{\text {cyc }}\right)[\pi] .
$$

Using Theorem 2.4 and Corollary 1.3, we deduce the following corollary:

Corollary 2.6. Let us keep the hypotheses and setting of Theorem 2.4. Then for an irreducible orthogonal representation $\sigma$ of $\Delta$, we get

$$
s_{p}\left(E_{1}, \sigma\right)+\sum_{v \in \Sigma_{0}} \delta_{E_{1}, v}(\sigma) \equiv s_{p}\left(E_{2}, \sigma\right)+\sum_{v \in \Sigma_{0}} \delta_{E_{2}, v}(\sigma) \quad(\bmod 2) .
$$

Further by Corollary 1.5 and Corollary 2.6, the comparison of parity of $s_{p}\left(E_{1}, \sigma\right)$ and $s_{p}\left(E_{2}, \sigma\right)$ reduces to comparing the parity of $\left\langle\sigma_{v}, \chi\right\rangle\left\langle\rho_{E_{i}, v}, \chi\right\rangle$ with $\chi \in \operatorname{Irr}_{\mathcal{F}}\left(\Delta_{v}\right)$, for each $v \in \Sigma_{0}$.

2.2. Comparison of the parity of $\delta_{E_{i}, v}(\sigma)$. In this subsection, we compare the parity of the local Iwasawa invariants $\delta_{E_{1}, v}(\sigma)$ with $\delta_{E_{2}, v}(\sigma)$ via the study of the quantity $\left\langle\sigma_{v}, \chi\right\rangle\left(\left\langle\rho_{E_{1}, v}, \chi\right\rangle-\left\langle\rho_{E_{2}, v}, \chi\right\rangle\right)$ (mod 2), according to the reduction type of $E_{1}$ and $E_{2}$ at each $v \in \Sigma_{0}$.

Lemma 2.7 (good - good). Let $v \nmid p$ be a prime of $F$ in $\Sigma_{0}$ such that $E_{1}$ and $E_{2}$ are good at $v$. Then $\delta_{E_{1}, v}(\sigma) \equiv \delta_{E_{2}, v}(\sigma)(\bmod 2)$.

Proof. $E_{1}$ and $E_{2}$ are good at $v$. Then using equation (19) in the relation (17), we only need to compute $\left\langle\rho_{E_{1}, v}, \chi\right\rangle-\left\langle\rho_{E_{2}, v}, \chi\right\rangle(\bmod 2)$ for $\chi \in\left\{\varphi_{E_{1}, v}, \psi_{E_{1}, v}, \varphi_{E_{2}, v}, \psi_{E_{2}, v}\right\}$. We know $V_{p}\left(E_{i}\right) \otimes_{\mathbb{Q}_{p}} \mathcal{F} \cong$ $\mathcal{F}\left(\varphi_{E_{i}, v}\right) \oplus \mathcal{F}\left(\psi_{E_{i}, v}\right)$ as $G_{F_{\text {cyc }, v}}$ modules. Also $E_{1}[p] \cong E_{2}[p]$ as $G_{\mathbb{Q}^{-}}$-modules and $\varphi_{E_{i}, v}, \psi_{E_{i}, v}$ are unramified characters of order prime to $p$. Thus we deduce $\left\{\varphi_{E_{1}, v}, \psi_{E_{1}, v}\right\}=\left\{\varphi_{E_{2}, v}, \psi_{E_{2}, v}\right\}$. Now using (17), $\delta_{E_{1}, v}(\sigma) \equiv \delta_{E_{2}, v}(\sigma)(\bmod 2)$. 
Lemma 2.8 (split - split). Let $v \in \Sigma_{0}$ be a prime of split multiplicative reduction for $E_{1}$ and $E_{2}$. Then $\delta_{E_{1}, v}(\sigma) \equiv \delta_{E_{2}, v}(\sigma)(\bmod 2)$.

Proof. In this case, using equation (21) in the relation (17), $\delta_{E_{i}, v}(\sigma) \equiv\left\langle\sigma_{v}, \omega_{v}\right\rangle(\bmod 2)$, which is the same for both $E_{1}$ and $E_{2}$. Thus $\delta_{E_{1}, v}(\sigma) \equiv \delta_{E_{2}, v}(\sigma)(\bmod 2)$.

Lemma 2.9 (non-split - non-split). Let $E_{1}$ and $E_{2}$ both have non-split multiplicative reduction at a $v \in \Sigma_{0}$. Then $\delta_{E_{1}, v}(\sigma) \equiv \delta_{E_{2}, v}(\sigma)(\bmod 2)$.

Proof. Recall $\varkappa_{v}$ is defined in (20). Again in this case, using equation (21) in the relation (17), $\delta_{E_{i}, v}(\sigma) \equiv$ $\left\langle\sigma_{v}, \omega_{v} \varkappa_{v}\right\rangle(\bmod 2)$, which is the same for both $E_{1}$ and $E_{2}$. Thus $\delta_{E_{1}, v}(\sigma) \equiv \delta_{E_{2}, v}(\sigma)(\bmod 2)$.

Lemma 2.10 (good - split). Let $v \in \Sigma_{0}$. Assume $E_{1}$ has good reduction at $v$ and $E_{2}$ has split multiplicative reduction at $v$, then $\delta_{E_{1}, v}(\sigma) \equiv \delta_{E_{2}, v}(\sigma)+\left\langle\sigma_{v}, 1_{v}\right\rangle(\bmod 2)$.

Proof. In this case, $\rho_{E_{1}, v} \sim\left(\begin{array}{cc}\varphi_{E_{1}, v} & 0 \\ 0 & \psi_{E_{1}, v}\end{array}\right)$ and $\rho_{E_{2}, v} \sim\left(\begin{array}{cc}\omega_{v} & * \\ 0 & 1\end{array}\right)$. Now $E_{1}[p] \cong E_{2}[p]$ implies that $\left\{\bar{\varphi}_{E_{1}, v}, \bar{\psi}_{E_{1}, v}\right\}=\left\{\bar{\omega}_{v}, \overline{1}_{v}\right\}=\left\{\omega_{v}, 1_{v}\right\}$. As $\varphi_{E_{1}, v}, \psi_{E_{1}, v}$ and $\omega_{v}$ have all orders prime to $p$, we see that $\left\{\varphi_{E_{1}, v}, \psi_{E_{1}, v}\right\}=\left\{\omega_{v}, 1_{v}\right\}$. Therefore, using (19) we obtain that $\delta_{E_{1}, v}(\sigma) \equiv\left\langle\sigma_{v}, \omega_{v}\right\rangle+\left\langle\sigma_{v}, 1_{v}\right\rangle(\bmod 2)$. On the other hand, by $(21), \delta_{E_{2}, v}(\sigma) \equiv\left\langle\sigma_{v}, \omega_{v}\right\rangle(\bmod 2)$. Hence, the result follows.

Lemma 2.11 (good - non-split). Let $v \in \Sigma_{0}$. Assume $E_{1}$ has good reduction at $v$ and $E_{2}$ has non-split multiplicative reduction at $v$, then $\delta_{E_{1}, v}(\sigma) \equiv \delta_{E_{2}, v}(\sigma)+\left\langle\sigma_{v}, \varkappa_{v}\right\rangle(\bmod 2)$.

Proof. The proof is similar to the good-split case in Lemma 2.10 and is omitted.

Lemma 2.12 (split - non-split). Let $v \in \Sigma_{0}$. Assume $E_{1}$ has split multiplicative reduction at $v$ and $E_{2}$ has non-split multiplicative reduction $v$. Then $\delta_{E_{1}, v}(\sigma) \equiv \delta_{E_{2}, v}(\sigma)+\left\langle\sigma_{v}, 1_{v}\right\rangle+\left\langle\sigma_{v}, \varkappa_{v}\right\rangle(\bmod 2)$.

Proof. By the proof of Lemma 2.8, $\delta_{E_{1}, v}(\sigma) \equiv\left\langle\sigma_{v}, \omega_{v}\right\rangle(\bmod 2)$. Similarly, by Lemma $2.9, \delta_{E_{2}, v}(\sigma) \equiv$ $\left\langle\sigma_{v}, \omega_{v} \varkappa_{v}\right\rangle(\bmod 2)$. Thus $\delta_{E_{1}, v}(\sigma) \equiv \delta_{E_{2}, v}(\sigma)+\left\langle\sigma_{v}, \omega_{v}\right\rangle+\left\langle\sigma_{v}, \omega_{v} \varkappa_{v}\right\rangle(\bmod 2)$.

By [AAS17, Lemma 2.8], the 3 conditions (i) $E_{1}$ is split at $v$, (ii) $E_{2}$ is non-split at $v$ and (iii) $E_{1}[p] \cong E_{2}[p]$, are simultaneously satisfied only when $\omega_{v}=\varkappa_{v}$. As $\varkappa_{v}$ is quadratic, the lemma is now immediate.

Lemma 2.13. If $E$ is potentially good at $v$, then $\rho_{E}\left(G_{F_{c y c, v}}\right)$ and $\bar{\rho}_{E}\left(G_{F_{c y c, v}}\right)$ are isomorphic.

Proof. By [ST68, Corollaries 2(b) \& 3], E acquires good reduction over $F_{\mathrm{cyc}, v}(E[p])$. Set $G:=\operatorname{Gal}\left(\bar{F}_{v} / F_{\mathrm{cyc}, v}(E[p])\right)$. Let $g \in G_{F_{\mathrm{cyc}, v}}$ be an element such that $\bar{\rho}_{E, v}(g)$ acts trivially on $E[p]$. Then, $g \in G$. Moreover, $G$ acts on $V_{p} E$ by the sum of two characters of order prime to $p$, after a suitable base change. Hence, $g$ acts trivially on $V_{p}(E)$ if and only if $\bar{\rho}_{E, v}(g)$ acts trivially on $E[p]$. This proves the lemma.

Lemma 2.14 (good - additive). Let $v \nmid p \infty$ be a prime of $F$. Then it is not possible that $E_{1}$ has good reduction while $E_{2}$ has additive reduction at $v$. In particular, this is true for any $v \in \Sigma_{0}$.

Proof. As $E_{1}$ is good at $v, E_{1}[p]$ is unramified. On the other hand, by Lemma 2.13, $E_{2}[p]$ is ramified. Since $E_{1}[p] \cong E_{2}[p]$, the lemma follows. 
Abbreviation: We write $E$ is $\mathrm{PMR}$ at a prime $v$ in $F$, if $E$ has additive and potentially multiplicative reduction at $v$. By $E$ is PGA (respectively PGNA) at a prime $v$ in $F$, we mean $E$ is additive, potentially good at $v$ with $\rho_{E}\left(G_{F_{\text {cyc }, v}}\right)$ abelian (respectively non-abelian).

Lemma 2.15 (multiplicative - additive). Let $v \in \Sigma_{0}$ be a multiplicative prime of $E_{1}$ and additive prime for $E_{2}$. Then by [AAS17, Lemma 2.5], it follows that $p=3$. Moreover, the following hold.

(i) It can not happen that $E_{1}$ is multiplicative at $v$ and $E_{2}$ is $P M R$ at $v$.

(ii) Assume that $E_{1}$ is split (resp. non-split) multiplicative at $v$ and $E_{2}$ is $P G A$ at $v$. Then $\mu_{3} \subset F_{v}$. If $v$ is split for $E_{1}$ then $\delta_{E_{1}, v}(\sigma) \equiv \delta_{E_{2}, v}(\sigma)+\left\langle\sigma_{v}, 1_{v}\right\rangle(\bmod 2)$ and if $v$ is non-split for $E_{1}$ then $\delta_{E_{1}, v}(\sigma) \equiv \delta_{E_{2}, v}(\sigma)+\left\langle\sigma_{v}, \varkappa_{v}\right\rangle(\bmod 2)$.

(iii) It is not possible that $E_{1}$ is multiplicative at $v$ and $E_{2}$ is PGNA at $v$, unless $3 \mid \# \rho_{E_{2}}\left(G_{F_{\mathrm{cyc}, v}}\right)$ and $\mu_{3} \not \subset F_{v}$. In the later case, $\delta_{E_{1}, v}(\sigma)+\left\langle\sigma_{v}, \chi_{v}\right\rangle \equiv \delta_{E_{2}, v}(\sigma)+\left\langle\sigma_{v}, \theta_{3, v}\right\rangle(\bmod 2)$, where $\theta_{3, v}$, is the unique 2-dimensional irreducible representation of $\operatorname{Gal}\left(F_{\mathrm{cyc}, v}\left(E_{2}[p]\right) / F_{\mathrm{cyc}, v}\right)$ which is the dihedral group $D_{6}$ of order 6 [Gre11, Page 165-166]. Here $\chi_{v}=\varkappa_{v}$ if $E_{1}$ is split at $v$ and $\chi_{v}=1_{v}$ if $E_{1}$ is non-split at $v$.

Proof. (i) If $E_{1}$ has multiplicative reduction at $v$, then the semi-simplification of $E_{1}[p]$ is unramified. On the other hand, if $E_{2}$ has PMR at $v$ then the semi-simplification of $E_{2}[p]$ is ramified. Thus, we cannot have this case.

(ii) Using (21) and $(24), \delta_{E_{1}, v}(\sigma) \equiv\left\langle\sigma_{v}, \omega_{v}\right\rangle(\bmod 2)$ if $E_{1}$ is split at $v, \delta_{E_{1}, v}(\sigma) \equiv\left\langle\sigma_{v}, \omega_{v} \varkappa_{v}\right\rangle$ $(\bmod 2)$ if $E_{1}$ is non-split at $v$ and $\delta_{E_{2}, v}(\sigma) \equiv\left\langle\sigma_{v}, \varepsilon_{E_{2}, v}\right\rangle+\left\langle\sigma_{v}, \varepsilon_{E_{2}, v}^{-1} \omega_{v}\right\rangle(\bmod 2)$.

Suppose that $3 \nmid \# \rho_{E_{2}}\left(G_{F_{\mathrm{cyc}, v}}\right)$. Then by Lemma $2.13,3 \nmid \# \bar{\rho}_{E_{2}}\left(G_{F_{\mathrm{cyc}, v}}\right)$. Since $\left.\bar{\rho}_{E_{1}}\right|_{I_{F_{v}}} \sim$ $\left(\begin{array}{ll}1 & * \\ 0 & 1\end{array}\right)$, we deduce that $E_{1}[p]$ is unramified and hence so is $E_{2}[p]$. This is a contradiction to the assumption that $E_{2}$ has potentially good additive reduction (see [ST68]).

Thus we are reduced to consider $3 \mid \# \rho_{E_{2}}\left(G_{F_{\mathrm{cyc}, v}}\right)$. Then $p=3$ and we have $\left.\bar{\rho}_{E_{1}}\right|_{I_{F_{v}}} \sim\left(\begin{array}{ll}1 & * \\ 0 & 1\end{array}\right)$ with $* \neq 0$. Also, $\left.\bar{\rho}_{E_{1}}\right|_{G_{F_{c y c}, v}} \sim\left(\begin{array}{cc}\omega_{v} & * \\ 0 & 1\end{array}\right)$. This implies that $\bar{\rho}_{E_{1}}\left(G_{F_{\mathrm{cyc}, v}}\right)$ is a non-abelian group if $\omega_{v}$ is non-trivial. Therefore $\bar{\rho}_{E_{1}}\left(G_{F_{\mathrm{cyc}, v}}\right)$ is also non-abelian. This is a contradiction. Therefore $\omega_{v}$ is trivial and hence we must have $\mu_{3} \subset F_{v}$. Since $\sigma_{v}$ is self dual, $\left\langle\sigma_{v}, \varepsilon_{E_{2}, v}\right\rangle=\left\langle\sigma_{v}, \varepsilon_{E_{2}, v}^{-1}\right\rangle$. Therefore $\delta_{E_{2}, v}(\sigma) \equiv 0(\bmod 2)$. This proves the claim.

(iii) Here $\delta_{E_{1}, v}(\sigma) \equiv\left\langle\sigma_{v}, \omega_{v}\right\rangle(\bmod 2)$ if $E_{1}$ is split at $v, \delta_{E_{1}, v}(\sigma) \equiv\left\langle\sigma_{v}, \omega_{v} \varkappa_{v}\right\rangle(\bmod 2)$ if $E_{1}$ is non-split at $v$ and $\delta_{E_{2}, v}(\sigma) \equiv\left\langle\sigma_{v}, \rho_{E_{2}, v}\right\rangle(\bmod 2)$. Note that for $p=3, \omega_{v}=\varkappa_{v}$ if $\mu_{3} \not \subset F_{v}$.

If $3 \nmid \# \rho_{E_{2}}\left(G_{F_{\mathrm{cyc}, v}}\right)$, then by the argument in part (ii) above, $E_{2}[p]$ is unramified and we get a contradiction. So, we can assume $3 \mid \# \bar{\rho}_{E_{2}}\left(G_{F_{\mathrm{cyc}, v}}\right)$. Further, $\mu_{3} \subset F_{\mathrm{cyc}, v}$ is also not possible; otherwise, $\bar{\rho}_{E_{1}}\left(G_{F_{\mathrm{cyc}, v}}\right)$ is abelian and by Lemma 2.13, $\rho_{E_{2}}\left(G_{F_{\mathrm{cyc}, v}}\right)$ is abelian as well.

Therefore we can assume $3 \mid \# \rho_{E_{2}}\left(G_{F_{\mathrm{cyc}, v}}\right)$ and $\mu_{3} \not \subset F_{\mathrm{cyc}, v}$. Then $\left.\bar{\rho}_{E_{1}}\right|_{G_{F_{c y c}, v}}$ is of order 6 and by Lemma 2.13, $\rho_{E_{2}}\left(G_{F_{\mathrm{cyc}, v}}\right) \cong \bar{\rho}_{E_{2}}\left(G_{F_{\mathrm{cyc}, v}}\right)$ is non-abelian. Consequently, $\rho_{E_{2}}\left(G_{F_{\mathrm{cyc}, v}}\right)$ is isomorphic to $D_{6}$. Let $\theta_{3, v}$ be the unique two dimensional irreducible representation of the dihedral group $\operatorname{Gal}\left(F_{c y c, v}\left(E_{2}[p]\right) / F_{\mathrm{cyc}, v}\right)$. We have that $\rho_{E_{2}, v} \sim \theta_{3, v}$. Thus the lemma follows. 
Lemma 2.16 (additive - additive). Let $v \in \Sigma_{0}$ be an additive prime for both $E_{1}$ and $E_{2}$. Then,

(i) If $E_{1}, E_{2}$ both are PMR at $v \in \Sigma_{0}$, then $\delta_{E_{1}, v}(\sigma) \equiv \delta_{E_{2}, v}(\sigma)(\bmod 2)$ if $\vartheta_{E_{1}, v}=\vartheta_{E_{2}, v}$, otherwise, $\delta_{E_{1}, v}(\sigma)+\left\langle\sigma_{v}, \vartheta_{E_{1}, v}\right\rangle \equiv \delta_{E_{2}, v}(\sigma)+\left\langle\sigma_{v}, \omega_{v} \vartheta_{E_{1}, v}\right\rangle(\bmod 2)$.

(ii) It can not happen that $E_{1}$ is PMR at $v$ while $E_{2}$ is PGNA at $v$, unless $p=3$, 3 divides $\# \rho_{E_{2}}\left(G_{F_{\mathrm{cyc}, v}}\right)$ and $\mu_{3} \not \subset F_{v}$. In the later case, $\delta_{E_{1}, v}(\sigma)+\left\langle\sigma_{v}, \omega_{v} \vartheta_{E_{1}, v}\right\rangle \equiv \delta_{E_{2}, v}(\sigma)+\left\langle\sigma_{v}, \theta_{3, v} \vartheta_{E_{1}, v}\right\rangle$ $(\bmod 2)$. Moreover, $\operatorname{Gal}\left(F_{\mathrm{cyc}, v}\left(E_{2}[p]\right) / F_{\mathrm{cyc}, v}\right)$ is the dihedral group of order 12 and it has a unique subgroup, say $H \cong D_{6}$. Then $\theta_{3, v}$ is the unique 2-dimensional irreducible representation of $H$.

(iii) If $E_{1}$ is $P M R$ at $v$ and $E_{2}$ is $P G A$ at $v$, then $\delta_{E_{1}, v}(\sigma) \equiv \delta_{E_{2}, v}(\sigma)+\left\langle\sigma_{v}, \vartheta_{E_{1}, v}\right\rangle(\bmod 2)$.

(iv) If $E_{1}, E_{2}$ are both $P G A$ at $v$, then $\delta_{E_{1}, v}(\sigma) \equiv \delta_{E_{2}, v}(\sigma)(\bmod 2)$.

(v) It is not possible that one of $E_{1}, E_{2}$ is PGA and the other one is PGNA at $v$.

(vi) Let $E_{1}, E_{2}$ be both PGNA at $v$. Then $\delta_{E_{1}, v}(\sigma) \equiv \delta_{E_{2}, v}(\sigma)(\bmod 2)$.

Proof. (i) Using (23), $\delta_{E_{i}, v}(\sigma) \equiv\left\langle\sigma_{v}, \omega_{v} \vartheta_{E_{i}, v}\right\rangle(\bmod 2)$. As $E_{1}[p] \cong E_{2}[p]$ and $\vartheta_{E_{1}, v}, \vartheta_{E_{2}, v}$ have orders prime to $p$, we deduce from (22) that either $\vartheta_{E_{1}, v}=\vartheta_{E_{2}, v}$ or $\vartheta_{E_{1}, v}=\omega_{v} \vartheta_{E_{2}, v}$ and the result follows. Note that $\vartheta_{E_{1}, v}=\omega_{v} \vartheta_{E_{2}, v}$ is possible only when $\omega_{v}$ is quadratic.

(ii) Using (23) and (24), $\delta_{E_{1}, v}(\sigma) \equiv\left\langle\sigma_{v}, \omega_{v} \vartheta_{E_{1}, v}\right\rangle(\bmod 2)$ and $\delta_{E_{2}, v}(\sigma) \equiv\left\langle\sigma_{v}, \rho_{E_{2}, v}\right\rangle(\bmod 2)$. Note that $E_{1}^{\prime}:=E_{1}^{\vartheta_{E_{1}, v}}$ has split multiplicative reduction at $v$. Also, since $\rho_{E_{2}, v}$ is absolutely irreducible, $E_{2}^{\prime}:=E_{2}^{\vartheta_{E_{1}, v}}$ is PGNA at $v$. Also, $E_{1}^{\prime}[p] \cong E_{2}^{\prime}[p]$.

First suppose that $p \nmid \# \rho_{E_{2}}\left(G_{F_{\mathrm{cyc}, v}}\right)$. Since $\vartheta_{E_{1}, v}$ is quadratic and $p$ is odd, it follows that $p \nmid \# \rho_{E_{2}^{\prime}}\left(G_{F_{\mathrm{cyc}, v}}\right)$. Now, by an argument similar to Lemma 2.15(iii), it is not possible that $E_{1}^{\prime}$ is split at $v, E_{2}^{\prime}$ is potentially good with $\rho_{E_{2}^{\prime}}\left(G_{F_{\text {cyc }, v}}\right)$ is non-abelian and $E_{1}^{\prime}[p] \cong E_{2}^{\prime}[p]$.

Hence, $p$ must divide $\# \rho_{E_{2}}\left(G_{F_{\text {cyc }, v}}\right)$. By the discussion after $(23)$, this can happen only when $p=3$. Also, as explained above, $p \mid \# \rho_{E_{2}^{\prime}}\left(G_{F_{\mathrm{cyc}, v}}\right)$. If $\mu_{3} \subset F_{v}$, then $\rho_{E_{2}^{\prime}}\left(G_{F_{\mathrm{cyc}, v}}\right)$ is abelian (see Lemma 2.15(iii)), which is not possible. So we can assume $\mu_{3} \not \subset F_{v}$. By Lemma 2.13, $3 \mid \# \bar{\rho}_{E_{2}^{\prime}}\left(G_{F_{\mathrm{cyc}, v}}\right)=\# \bar{\rho}_{E_{1}^{\prime}}\left(G_{F_{\mathrm{cyc}, v}}\right)$. Using these facts in the expression $\left.\bar{\rho}_{E_{1}^{\prime}}\right|_{G_{F_{\mathrm{cyc}, v}}} \sim\left(\begin{array}{cc}\omega_{v} & * \\ 0 & 1\end{array}\right)$, we obtain that $\# \bar{\rho}_{E_{1}^{\prime}}\left(G_{F_{\mathrm{cyc}, v}}\right)=6=\# \bar{\rho}_{E_{2}^{\prime}}\left(G_{F_{\mathrm{cyc}, v}}\right)$. Thus, by Lemma 2.13, $\rho_{E_{2}^{\prime}}\left(G_{F_{\mathrm{cyc}, v}}\right)$ is non-abelian of order 6 . It follows that $\rho_{E_{2}^{\prime}, v} \cong \theta_{3, v}$ and $\rho_{E_{2}, v} \cong \theta_{3, v} \vartheta_{E_{1}, v}$. The result follows.

(iii) Using (23) and $(24), \delta_{E_{1}, v}(\sigma) \equiv\left\langle\sigma_{v}, \omega_{v} \vartheta_{E_{1}, v}\right\rangle(\bmod 2)$ and $\delta_{E_{2}, v}(\sigma) \equiv\left\langle\sigma_{v}, \varepsilon_{E_{2}, v}\right\rangle+\left\langle\sigma_{v}, \omega_{v} \varepsilon_{E_{2}, v}^{-1}\right\rangle$ $(\bmod 2)$. Set $E_{1}^{\prime}:=E_{1}^{\vartheta^{\prime} E_{1}, v}$ and $E_{2}^{\prime}:=E_{2}^{\vartheta E_{1}, v}$. Then $E_{1}^{\prime}$ is split multiplicative at $v, E_{2}^{\prime}$ is PGA at $v$ and $E_{1}^{\prime}[p] \cong E_{2}^{\prime}[p]$.

If possible, let $p \nmid \# \rho_{E_{2}}\left(G_{F_{\mathrm{cyc}, v}}\right)$. Then as explained in $\operatorname{part}(\mathrm{ii}), p \nmid \# \rho_{E_{2}^{\prime}}\left(G_{F_{\mathrm{cyc}, v}}\right)$. Now, since $E_{1}^{\prime}[p] \cong E_{2}^{\prime}[p]$, this is not possible by the proof of Lemma 2.15(ii).

Thus, $p$ must divide $\# \rho_{E_{2}}\left(G_{F_{\mathrm{cyc}, v}}\right)$ and hence $p \mid \# \rho_{E_{2}^{\prime}}\left(G_{F_{\mathrm{cyc}, v}}\right)$. This can only happen at $p=3$ (see the discussion after (23)). Further, using Lemma 2.15(ii), $\mu_{3} \subset F_{\mathrm{cyc}, v}$ and $\delta_{E_{2}, v}(\sigma) \equiv 0$ $(\bmod 2)$. So we arrive at the result.

(iv) Using (24), $\delta_{E_{i}, v}(\sigma) \equiv\left\langle\sigma_{v}, \varepsilon_{E_{i}, v}\right\rangle+\left\langle\sigma_{v}, \omega_{v} \varepsilon_{E_{i}, v}^{-1}\right\rangle(\bmod 2)$. When $p \nmid \# \rho_{E_{1}}\left(G_{F_{\mathrm{cyc}, v}}\right) \# \rho_{E_{2}}\left(G_{F_{\mathrm{cyc}, v}}\right)$, the proof is similar to Lemma 2.7 and is omitted. 
Assume that $p$ divides the order of $\rho_{E_{i}}\left(G_{F_{\mathrm{cyc}, v}}\right)$ for either $i=1$ or $i=2$. Then we necessarily have $p=3$. Also for that $i, \varepsilon_{E_{i}, v}$ has order divisible by 3 . Then $F_{\mathrm{cyc}, v}$ will have a cyclic $p$ extension with ramification index divisible by 3 and by local class field theory, $\mu_{3} \subset F_{\text {cyc }, v}$. As a result, $\omega_{v}=1_{v}$. Since $\sigma_{v}$ is self-dual, $\delta_{E_{2}, v}(\sigma) \equiv\left\langle\sigma_{v}, \varepsilon_{E_{2}, v}\right\rangle+\left\langle\sigma_{v}, \varepsilon_{E_{2}, v}^{-1}\right\rangle \equiv 0(\bmod 2)$.

(v) By Lemma 2.13, $\bar{\rho}_{E_{1}}\left(G_{F_{\text {cyc }, v}}\right)$ is abelian and $\bar{\rho}_{E_{2}}\left(G_{F_{\text {cyc }, v}}\right)$ is non-abelian but they are isomorphic. This is not possible.

(vi) Again recall, $\rho_{E_{i}}\left(G_{F_{\mathrm{cyc}, v}}\right) \cong \bar{\rho}_{E_{i}}\left(G_{F_{\mathrm{cyc}, v}}\right)$ by Lemma 2.13. Note that $\rho_{E_{i}, v}$ is absolutely irreducible. From [Ser72, Page 312], the image $\rho_{E_{i}}\left(I_{F_{v}}\right)$ is

- a cyclic group of order $2,3,4$ or 6 , if $v \nmid 6$

- a cyclic group of order $2,3,4,6$ or a semi-direct product $\mathbb{Z} / 3 \mathbb{Z} \rtimes \mathbb{Z} / 4 \mathbb{Z}$, if $v \mid 3$

- a subgroup of $\mathrm{SL}_{2}\left(\mathbb{F}_{3}\right)$ of order $2,3,4,6,8$ or 24 , if $v \mid 2$.

First consider the case that $p \nmid \# \rho\left(G_{F_{\mathrm{cyc}, v}}\right)$. When $p \nmid \# \rho\left(G_{F_{\mathrm{cyc}, v}}\right)$, the reduction $\bmod p$ induces a bijection between the set of irreducible representations $\rho$ of $G_{F_{\mathrm{cyc}, v}}$ and the set of all irreducible representations $\bar{\rho}$ of $G_{F_{\mathrm{cyc}, v}}$ (see for instance [Wei03, P. 85-86]). Using this fact together with $\bar{\rho}_{E_{1}, v} \cong \bar{\rho}_{E_{2}, v}$, we deduce that $\rho_{E_{1}, v} \cong \rho_{E_{2}, v}$. Now from $(24), \delta_{E_{i}, v}(\sigma) \equiv\left\langle\sigma_{v}, \rho_{E_{i}, v}\right\rangle$ $(\bmod 2)$. Therefore, $\delta_{E_{1}, v}(\sigma) \equiv \delta_{E_{2}, v}(\sigma)(\bmod 2)$.

In the second case, when $p \mid \# \rho\left(G_{F_{\mathrm{cyc}, v}}\right)$, again recall that $p=3$ and hence $v \nmid 3$. Thus the possibilities we have that $v \mid 2$ and $\# \rho_{E_{i}}\left(I_{F_{v}}\right) \in\{3,6,24\}$ or $v \nmid 6$ and $\# \rho_{E_{i}}\left(I_{F_{v}}\right) \in\{3,6\}$.

Let us consider the subcase when $\# \rho_{E_{i}}\left(I_{F_{v}}\right)=3$ for $i=1$, 2 . As $\rho_{E_{i}}\left(I_{F_{v}}\right)$ is cyclic of order 3 and the image of $\rho_{E_{i}, v}$ is non-abelian, $\rho_{E_{i}}\left(G_{F_{\mathrm{cyc}, v}}\right) \cong \rho_{E_{i}}\left(I_{F_{v}}\right) \rtimes \mathbb{Z} / 2 \mathbb{Z} \cong D_{6}$ (see [Roh94, Page 153]). Since there is a unique 2-dimensional irreducible representation of $D_{6}$, we deduce $\rho_{E_{1}, v} \cong \rho_{E_{2}, v}$. Therefore, $\delta_{E_{1}, v}(\sigma) \equiv \delta_{E_{2}, v}(\sigma)(\bmod 2)$.

Next subcase is when $\# \rho_{E_{i}}\left(I_{F_{v}}\right)=6$ for $i=1,2$. As $\mathrm{SL}_{2}\left(\mathbb{F}_{3}\right)$ has no subgroup isomorphic to $D_{6}, \rho_{E_{i}}\left(I_{F_{v}}\right) \cong \mathbb{Z} / 6 \mathbb{Z}$. Once again, by using arguments of [Roh94, Page 153], we obtain the structure $\rho_{E_{i}}\left(G_{F_{\text {cyc }, v}}\right) \cong \rho_{E_{i}}\left(I_{F_{v}}\right) \rtimes \mathbb{Z} / 2 \mathbb{Z} \cong D_{12}$. Since there is only one faithful 2-dimensional irreducible representation of $D_{12}$, as observed from the character table of $D_{12}$, we conclude that $\rho_{E_{1}, v} \cong \rho_{E_{2}, v}$. Hence, $\delta_{E_{1}, v}(\sigma) \equiv \delta_{E_{2}, v}(\sigma)(\bmod 2)$.

Finally, we are left to deal with the subcase where $p=3$ and $\rho_{E_{i}}\left(I_{F_{v}}\right) \cong \mathrm{SL}_{2}\left(\mathbb{F}_{3}\right)$ of order 24. Then $v \mid 2$. Also notice that, as Weil pairing is non-degenerate, $\mu_{3} \subset F_{v}$ if and only if $\rho_{E_{i}}\left(G_{F_{\mathrm{cyc}, v}}\right) \subset \mathrm{SL}_{2}\left(\mathbb{F}_{3}\right)$. Hence if $\mu_{3} \subset F_{v}$, then $\rho_{E_{i}, v}$ is self-dual and $\rho_{E_{i}}\left(G_{F_{\mathrm{cyc}, v}}\right) \cong \mathrm{SL}_{2}\left(\mathbb{F}_{3}\right)$ for $i=1,2$. From the character table, there is only one 2 -dimensional irreducible representation self dual representation of $\mathrm{SL}_{2}\left(\mathbb{F}_{3}\right)$. Hence, $\rho_{E_{i}, v}$ is uniquely determined and we deduce $\rho_{E_{1}, v} \cong \rho_{E_{2}, v}$. On the other hand, if $\mu_{3} \not \subset F_{v}$, then $\rho_{E_{i}}\left(G_{F_{\mathrm{cyc}, v}}\right) \cong \mathrm{GL}_{2}\left(\mathbb{F}_{3}\right)$ for $i=1,2$. From the character table, $\mathrm{GL}_{2}\left(\mathbb{F}_{3}\right)$ has three 2-dimensional irreducible representations and further, all three of these 2 dimensional representations have distinct traces $(\bmod 3)$. Since $\left.\left.\bar{\rho}_{E_{1}}\right|_{G_{F_{\mathrm{cyc}, ~}}} \cong \bar{\rho}_{E_{2}}\right|_{G_{F_{\mathrm{cyc}, v}}}$, we deduce $\rho_{E_{1}, v} \cong \rho_{E_{2}, v}$. So $\delta_{E_{1}, v}(\sigma) \equiv \delta_{E_{2}, v}(\sigma)(\bmod 2)$.

2.3. The main theorem on the algebraic side. Let $B$ be the set of primes of $F$, such that both $E_{1}$ and $E_{2}$ have additive reduction and at least one of them has PMR. At each $v \in B$, we define a quadratic character $\vartheta_{v}$ : if $E_{1}$ (respectively $E_{2}$ ) has PMR at $v$ and $E_{2}$ (respectively $E_{1}$ ) has potentially good reduction then put $\vartheta_{v}:=\vartheta_{E_{1}, v}\left(\right.$ respectively $\left.\vartheta_{E_{2}, v}\right)$. When both $E_{1}$ and $E_{2}$ have PMR at $v$, put 
$\vartheta_{v}:=\vartheta_{E_{1}, v}$. Note, in this last case by Lemma 2.16(i), either $\vartheta_{E_{1}, v}=\vartheta_{E_{2}, v}$ or $\vartheta_{E_{1}, v}=\omega_{v} \vartheta_{E_{2}, v}$ with $\omega_{v}$ quadratic. The table below summarises the cases where $\delta_{E_{1}, v}(\sigma)$ and $\delta_{E_{2}, v}(\sigma)$ can have different parity. In the following table, we use the notation $a \equiv_{2} b$ to denote $a \equiv b(\bmod 2)$.

\begin{tabular}{|l|l|l|}
\hline $\mathbf{p}, \mathbf{v} \nmid \mathbf{p}$ & \multicolumn{1}{|c|}{ Reduction type at $v$} & \multicolumn{1}{c|}{ Parity } \\
\hline$p \geq 3$ & $E_{1}$ is good and $E_{2}$ is split & $\delta_{E_{1}, v}(\sigma) \equiv_{2} \delta_{E_{2}, v}(\sigma)+\left\langle\sigma_{v}, 1_{v}\right\rangle$ \\
\hline$p \geq 3$ & $E_{1}$ is good and $E_{2}$ is non-split & $\delta_{E_{1}, v}(\sigma) \equiv_{2} \delta_{E_{2}, v}(\sigma)+\left\langle\sigma_{v}, \varkappa_{v}\right\rangle$ \\
\hline$p \geq 3$ & $E_{1}$ is split and $E_{2}$ is non-split & $\delta_{E_{1}, v}(\sigma) \equiv_{2} \delta_{E_{2}, v}(\sigma)+\left\langle\sigma_{v}, 1_{v}\right\rangle+\left\langle\sigma_{v}, \varkappa_{v}\right\rangle$ \\
\hline$p \geq 3$ & $E_{1}, E_{2}$ are PMR and $\vartheta_{E_{1}, v} \neq \vartheta_{E_{2}, v}$ & $\delta_{E_{1}, v}(\sigma)+\left\langle\sigma_{v}, \vartheta_{E_{1}, v}\right\rangle \equiv_{2} \delta_{E_{2}, v}(\sigma)+\left\langle\sigma_{v}, \omega_{v} \vartheta_{E_{1}, v}\right\rangle$ \\
\hline$p \geq 3$ & $E_{1}$ is PMR and $E_{2}$ is PGA & $\delta_{E_{1}, v}(\sigma) \equiv_{2} \delta_{E_{2}, v}(\sigma)+\left\langle\sigma_{v}, \vartheta_{v}\right\rangle$ \\
\hline$p=3, \mu_{3} \subset F_{v}$ & $E_{1}$ is split and $E_{2}$ is PGA & $\delta_{E_{1}, v}(\sigma) \equiv_{2} \delta_{E_{2}, v}(\sigma)+\left\langle\sigma_{v}, 1_{v}\right\rangle$ \\
\hline$p=3, \mu_{3} \subset F_{v}$ & $E_{1}$ is non-split and $E_{2}$ is PGA & $\delta_{E_{1}, v}(\sigma) \equiv_{2} \delta_{E_{2}, v}(\sigma)+\left\langle\sigma_{v}, \varkappa_{v}\right\rangle$ \\
\hline$p=3, \mu_{3} \not \subset F_{v}$ & $E_{1}$ is split and $E_{2}$ is PGNA & $\delta_{E_{1}, v}(\sigma)+\left\langle\sigma_{v}, \varkappa_{v}\right\rangle \equiv_{2} \delta_{E_{2}, v}(\sigma)+\left\langle\sigma_{v}, \theta_{3, v}\right\rangle$ \\
\hline$p=3, \mu_{3} \not \subset F_{v}$ & $E_{1}$ is non-split and $E_{2}$ is PGNA & $\delta_{E_{1}, v}(\sigma)+\left\langle\sigma_{v}, 1_{v}\right\rangle \equiv_{2} \delta_{E_{2}, v}(\sigma)+\left\langle\sigma_{v}, \theta_{3, v}\right\rangle$ \\
\hline
\end{tabular}

TABLE 1. Parity of $\delta_{E_{i}, v}(\sigma)$

Definition 2.17. Let $S_{i}, N_{i}$ respectively denote the set of primes $v \in \Sigma_{0}$ such that the elliptic curve $E_{i} / F$ has split multiplicative, respectively non-split multiplicative reduction at $v$. Let $\Sigma_{0}^{\prime}$ denote the set the primes in $\Sigma_{0}$ where $\sigma$ is ramified.

$W=\left\{v \in \Sigma_{0}^{\prime}: \mu_{p} \not \subset F_{v}\right.$, both $E_{1}, E_{2}$ are PMR at $v$ and $\left.\vartheta_{E_{1}, v} \neq \vartheta_{E_{2}, v}\right\}$.

$X=\left\{v \in \Sigma_{0}^{\prime}:\right.$ one of $E_{1}, E_{2}$ is PMR at $v$ and the other is PGA at $\left.v\right\}$.

$Y_{3}=\left\{v \in \Sigma_{0}: p=3, \mu_{3} \not \subset F_{v}\right.$, one of $E_{1}, E_{2}$ is multiplicative at $v$ and the other is PGNA at $\left.v\right\}$.

$Z_{3}=\left\{v \in \Sigma_{0}^{\prime}: p=3, \mu_{3} \not \subset F_{v}\right.$, one of $E_{1}, E_{2}$ is PMR at $v$ and the other is PGNA at $\left.v\right\}$.

Using Corollary 2.6 and combining Lemmas 2.7 to 2.15, we obtain the following theorem:

Theorem 2.18. Let $p$ be an odd prime and $k$ be a number field such that all primes of $k$ lying above $p$ are ramified in $k\left(\mu_{p}\right) / k$. Let $E_{1}, E_{2}$ be two elliptic curves over $k$ with $E_{1}[p] \cong E_{2}[p]$ as $G_{k}$-modules. Let $\sigma$ be an absolutely irreducible self-dual orthogonal representation of $\Delta$. Assume the hypothesis $(\mathbf{H})$. 
Then

$$
\begin{aligned}
& s_{p}\left(E_{1}, \sigma\right)+\sum_{v \in S_{1}}\left\langle\sigma_{v}, 1_{v}\right\rangle+\sum_{v \in N_{1}}\left\langle\sigma_{v}, \varkappa_{v}\right\rangle+\sum_{v \in W}\left(\left\langle\sigma_{v}, \vartheta_{v}\right\rangle+\left\langle\sigma_{v}, \omega_{v} \vartheta_{v}\right\rangle\right)+\sum_{v \in Z_{3}}\left(\left\langle\sigma_{v}, \omega_{v} \vartheta_{v}\right\rangle+\left\langle\sigma_{v}, \vartheta_{v} \theta_{3, v}\right\rangle\right) \\
& \equiv s_{p}\left(E_{2}, \sigma\right)+\sum_{v \in S_{2}}\left\langle\sigma_{v}, 1_{v}\right\rangle+\sum_{v \in N_{2}}\left\langle\sigma_{v}, \varkappa_{v}\right\rangle+\sum_{v \in X}\left\langle\sigma_{v}, \vartheta_{v}\right\rangle+\sum_{v \in Y_{3}}\left(\left\langle\sigma_{v}, 1_{v}\right\rangle+\left\langle\sigma_{v}, \varkappa_{v}\right\rangle+\left\langle\sigma_{v}, \theta_{3, v}\right\rangle\right) \quad(\bmod 2) .
\end{aligned}
$$

Corollary 2.19. We keep the hypotheses and setting of Theorem 2.18. In addition, whenever $v$ is a prime of additive reduction for at least one of $E_{1}, E_{2}$, we assume that $\mu_{p} \subset F_{v}$. Then

$s_{p}\left(E_{1}, \sigma\right)+\sum_{v \in S_{1}}\left\langle\sigma_{v}, 1_{v}\right\rangle+\sum_{v \in N_{1}}\left\langle\sigma_{v}, \varkappa_{v}\right\rangle \equiv s_{p}\left(E_{2}, \sigma\right)+\sum_{v \in S_{2}}\left\langle\sigma_{v}, 1_{v}\right\rangle+\sum_{v \in N_{2}}\left\langle\sigma_{v}, \varkappa_{v}\right\rangle+\sum_{v \in X}\left\langle\sigma_{v}, \vartheta_{v}\right\rangle \quad(\bmod 2)$.

Remark 2.20. From the definitions in (20) and (22), it is clear that $\varkappa_{v}$ and $\vartheta_{v}$ can be considered as characters of $G_{F_{v}}$ as well. Now, let $E_{1}$ is split mult. at $v$ and $E_{2}$ is PGNA at $v$. Then, necessarily $p=3$ and $\operatorname{Gal}\left(F_{\text {cyc }, v}\left(E_{1}[p]\right) / F_{\text {cyc }, v}\right) \cong D_{6}$ (see Lemma 2.15(iii)). Further, $\left.\bar{\rho}_{E_{1}}\right|_{G_{F_{v}}} \sim$ $\left(\begin{array}{cc}\chi_{1} & * \\ 0 & \chi_{2}\end{array}\right)$ with $\left\{\chi_{1}, \chi_{2}\right\}=\left\{\omega_{v}, 1_{v}\right\}$ and $\omega_{v} \neq 1_{v}$. Hence, $\operatorname{Gal}\left(F_{v}\left(E_{1}[p]\right) / F_{v}\right)$ is also the non-abelian of order 6, i.e., $\operatorname{Gal}\left(F_{v}\left(E_{1}[p]\right) / F_{v}\right) \cong \operatorname{Gal}\left(F_{\text {cyc }, v}\left(E_{1}[p]\right) / F_{\text {cyc }, v}\right)$ and $F_{v}\left(E_{1}[p]\right) \cap F_{\text {cyc }, v}=F_{v}$. In fact, $\operatorname{Gal}\left(F_{\text {cyc }, v}\left(E_{1}[p]\right) / F_{v}\right) \cong \operatorname{Gal}\left(F_{\text {cyc }, v}\left(E_{1}[p]\right) / F_{\text {cyc }, v}\right) \times \operatorname{Gal}\left(F_{\text {cyc }, v} / F_{v}\right)$. Thus we may as well consider $\theta_{3, v}$ to be the unique irreducible representation of degree two of $\operatorname{Gal}\left(F_{v}\left(E_{1}[p]\right) / F_{v}\right) \cong \operatorname{Gal}\left(F_{v}\left(E_{2}[p]\right) / F_{v}\right)$.

Let $\tau_{v}$ be a sub-representation of $\left.\sigma\right|_{G_{F_{v}}}$ such that $\left.\tau_{v}\right|_{G_{F_{\mathrm{cyc}, v}}}$ is equivalent to $\left.\theta_{3, v}\right|_{G_{F_{\mathrm{cyc}, v}}}$. Then $\left.\left.\tau_{v}\right|_{G_{F v}} \sim \theta_{3, v}\right|_{G_{F v}} \otimes \alpha$ for a character $\alpha$ of $\operatorname{Gal}\left(F_{\text {cyc }, v} / F_{v}\right)$ of $p$-power order. Since $\left.\sigma\right|_{G_{F v}}$ is self-dual and $p$ is odd, $\left\langle\left.\sigma\right|_{G_{F_{v}}},\left.\theta_{3, v}\right|_{G_{F_{v}}} \otimes \alpha,\right\rangle=\left\langle\left.\sigma\right|_{G_{F_{v}}},\left.\theta_{3, v}\right|_{G_{F_{v}}} \otimes \alpha^{-1},\right\rangle$. This shows that $\left\langle\left.\sigma\right|_{G_{F_{v}}},\left.\theta_{3, v}\right|_{G_{F_{v}}}\right\rangle=$

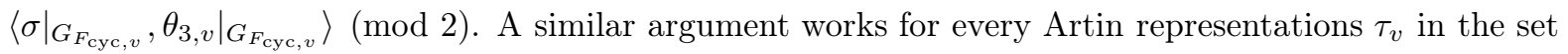
$C=\left\{\varkappa_{v}, 1_{v}, \vartheta_{v}, \vartheta_{v} \theta_{3, v}, \theta_{3, v}\right\}$. Thus the multiplicities $\left\langle\sigma_{v}, \dagger>, \dagger \in C\right.$ in Theorem 2.18 can be considered over $G_{F_{v}}$ as well.

This observation will be used in $\S 3$ to compare these multiplicities with the local root numbers over $F_{v}$.

\section{Relation With the Root numbers}

3.1. Basic results on root number. Let $k, F, K$ and $E \in\left\{E_{1}, E_{2}\right\}$ be as before. Recall $\sigma: \Delta=$ $\operatorname{Gal}(K / F) \rightarrow \mathrm{GL}_{n}(\mathcal{F})$ is a self-dual Artin representation. Also, $O$ is the ring of integer of $\mathcal{F}$ with uniformizer $\pi$. In view of Remark 2.20, we now use the notation $\sigma_{v}:=\left.\sigma\right|_{G_{F v}}$. For a prime $v$ of $F$, let (i) $\rho_{E / F_{v}}^{\prime}$, (ii) $\rho_{E / F_{v}}$ and (iii) $\rho_{E, v}=\left.\rho_{E}\right|_{G_{F_{\mathrm{cyc}, v}}}$ respectively denote (i) the representations of the Weil-Deligne group $\mathcal{W}^{\prime}\left(F_{v}\right)$ of $F_{v}$ associated to $E$, (ii) the Weil group $\mathcal{W}\left(F_{v}\right)$ of $F_{v}$ associated to $E$ $\left[\right.$ Roh94, §2, §3] and (iii) the restriction of the $p$-adic Galois representation $\rho_{E}: G_{k} \rightarrow \operatorname{Aut}\left(V_{p} E\right)($ see $\S 1)$ at $G_{F_{\mathrm{cyc}, v}}$.

We will denote by $V_{E}$ the representation space for $\rho_{E / F_{v}}^{\prime}$. Set $V_{E}^{\sigma}:=V_{E} \otimes \sigma$. Let $\varepsilon\left(V_{E}^{\sigma}\right)$ denote the local epsilon factor associated to $V_{E}^{\sigma}$ at $v$ [Del73]. The local root number, $W\left(E / F_{v}, \sigma_{v}\right)$, at $v$ is then given by [Roh96]

$$
W\left(E / F_{v}, \sigma_{v}\right)=\operatorname{sign}\left(\varepsilon\left(V_{E}^{\sigma}\right)\right)=\frac{\varepsilon\left(V_{E}^{\sigma}\right)}{\left|\varepsilon\left(V_{E}^{\sigma}\right)\right|} .
$$

Since $\sigma$ is self dual, $W\left(E / F_{v}, \sigma_{v}\right) \in\{ \pm 1\}$ [Roh96, Equ. (3.1)]. The global root number $W(E / F, \sigma)$, is a product of all local root numbers i.e. $W(E / F, \sigma):=\prod_{\text {all prime } v} W\left(E / F_{v}, \sigma_{v}\right)$ [Roh96]. 
Note that the local Artin reciprocity map carries $F_{v}^{\times}$isomorphically onto $\mathcal{W}\left(F_{v}\right)^{\text {ab }}$ inside $\operatorname{Gal}\left(F_{v}^{a b} / F_{v}\right)$. In particular, if $\phi$ is a character of $G_{F_{v}}$, then $\phi(-1)$ is well defined and again by the local reciprocity law, $\phi(-1)=1$ if $\phi$ is unramified (see for example, [Roh94, §1]). Let $\omega(v)$ denote the unramified character of $\mathcal{W}\left(F_{v}\right)$ sending a Frobenius element to $q_{v}$, the order of the residue field of $F_{v}$. We recall the following results of Rohrlich which will be used to compare the local root numbers of $E_{1}$ and $E_{2}$.

Theorem 3.1. Let $E / F$ be an elliptic curve, $\sigma$ be a self-dual, irreducible representation of $\Delta$.

(i) [Roh96, Theorem 2(i)] If $v$ is an archimedean prime of $F$, then $W\left(E / F_{v}, \sigma_{v}\right)=(-1)^{\operatorname{dim} \sigma}$.

(ii) [Roh96, Prop. 8(i)] If $E$ has good reduction at a finite prime $v$ of $F$, then $W\left(E / F_{v}, \sigma_{v}\right)=$ $\operatorname{det} \sigma_{v}(-1)$.

(iii) [Roh96, Theorem 2(ii)] If $\operatorname{ord}_{v}\left(j_{E}\right)<0$, then $W\left(E / F_{v}, \sigma_{v}\right)=\operatorname{det} \sigma_{v}(-1) \phi_{v}(-1)^{\operatorname{dim} \sigma}(-1)^{\left\langle\sigma_{v}, \phi_{v}\right\rangle}$. Here $\phi_{v}=1_{v}$ if $v$ is split multiplicative, $\phi_{v}=\varkappa_{v}$ is the unramified quadratic character if $v$ is non-split multiplicative, and $\phi_{v}=\vartheta_{E, v}($ see (22)) is ramified quadratic if $E$ is PMR at $v$.

(iv) Let $v$ be a prime of additive, potentially good reduction of $E$.

(a) [Roh08, Prop. 2] If $E$ is PGA at $v$, then $\rho_{E / F_{v}} \otimes \omega(v)^{1 / 2} \cong \chi_{E, v} \oplus \chi_{E, v}^{-1}$, for some character $\chi_{v}$ of $F_{v}^{\times}$. In this case [Roh08, (1.6)], $W\left(E / F_{v}, \sigma_{v}\right)=\operatorname{det} \sigma_{v}(-1) \chi_{E, v}(-1)^{\operatorname{dim} \sigma}$.

(b) Let $E$ be PGNA at $v$. If $v \nmid 6$, then by the proof of [Roh96, Theorem 2(iii), page 332], $\rho_{E_{2} / F_{v}} \otimes \omega(v)^{1 / 2}=\operatorname{Ind}_{L / F_{v}} \phi$, where $L / F_{v}$ is an unramified quadratic extension and $\phi$ is a character of $L^{\times}$. Write $L=F_{v}(u)$, for some $u \in O_{L}^{\times}$. Further by [Roh96, Theorem 1], $W\left(E / F_{v}, \sigma_{v}\right)=\operatorname{det} \sigma_{v}(-1) \phi(u)^{\operatorname{dim} \sigma}(-1)^{\left\langle\sigma_{v}, 1_{v}\right\rangle+\left\langle\sigma_{v}, \varkappa_{v}\right\rangle+\left\langle\sigma_{v}, \theta_{e, v}\right\rangle}$, where $e:=\# \rho_{E}\left(I_{F_{v}}\right)=$ $\# \bar{\rho}_{E}\left(I_{F_{v}}\right)$ and $\theta_{e, v}$ is the faithful 2-dimensional representation of $\operatorname{Gal}\left(F_{v}(E[p]) / F_{v}\right) \cong D_{2 e}$.

Remark 3.2. (1) Recall, $\rho_{E / F_{v}}^{\prime}$ is a pair $\left(\rho_{E / F_{v}}, N\right)$, where $N$ is a nilpotent endomorphism on $V_{E}$ satisfying $\rho_{E / F_{v}}(g) N \rho_{E / F_{v}}(g)^{-1}=\omega(v)(g) N$ for all $g \in \mathcal{W}\left(F_{v}\right)$ [Roh94, §3]. When $E$ is additive and potentially good at $v \mid \ell$, with $\ell \neq p$ then $N=0$ and $\rho_{E / F_{v}}^{\prime}=\rho_{E / F_{v}}$ [Roh94, Prop. §14].

In the case (iv)(a) of Theorem 3.1, we have $\rho_{E, v} \cong \varepsilon_{E, v} \oplus \omega_{v} \varepsilon_{E, v}^{-1}$ (cf. $\S 1$ ). As any real power of $\omega(v)$ is unramified, we see from the definition of a representation of Weil-Deligne group [Roh94, Prop. §4] that $\varepsilon_{E, v}(-1)=\chi_{E, v}(-1)$.

(2) In Theorem 3.1 (iv)(b), if $v \mid 2$ or $v \mid 3$, we see from the proof of [Roh96, Theorem 2(iii)] that as long as $\rho_{E / F_{v}}$ is an induced representation from an unramified quadratic extension, we can continue to use the formula in Theorem 3.1 (iv)(b).

3.2. Comparison of the root numbers. Note that $\rho_{E_{i} / F_{v}}^{\prime} \otimes \omega(v)^{1 / 2}$ is symplectic [Roh94, §16]. Let $a\left(\rho_{E / F_{v}}^{\prime}\right)$ denote the conductor exponent of $\rho_{E / F_{v}}^{\prime}$ (see [Roh94, §10]). At first, we prove the following:

Lemma 3.3. Let $E_{1}, E_{2}$ be two elliptic curves with $E_{1}[p] \cong E_{2}[p]$ as $G_{k}$-modules. Let $v \mid \ell$ with $\ell \neq p$ be a finite prime of $F$ such that $a\left(\rho_{E_{1} / F_{v}}^{\prime} \otimes \sigma\right)=a\left(\rho_{E_{2} / F_{v}}^{\prime} \otimes \sigma\right)$. Then

$$
\frac{W\left(E_{1} / F_{v}, \sigma_{v}\right)}{W\left(E_{2} / F_{v}, \sigma_{v}\right)}=\frac{\operatorname{det}\left(- \text { Frob }_{\mathrm{cyc}, v} \mid\left(V_{E_{2}}^{\sigma}\right)^{I_{F v}}\right)}{\operatorname{det}\left(- \text { Frob }_{\mathrm{cyc}, v} \mid\left(V_{E_{1}}^{\sigma}\right)^{I_{F v}}\right)} \quad(\bmod \pi) .
$$


Proof. Let $E \in\left\{E_{1}, E_{2}\right\}$. For $v \mid \ell$ with $\ell \neq p$, recall the modified local constant, $\varepsilon_{0}\left(V_{E}^{\sigma}\right)$, defined by Deligne [Del73, Equ. (5.1) \& Theorem 6.5(a)]:

$$
\varepsilon_{0}\left(V_{E}^{\sigma}\right)=\varepsilon\left(V_{E}^{\sigma}\right) \operatorname{det}\left(-\operatorname{Frob}_{v} \mid\left(V_{E}^{\sigma}\right)^{I_{F_{v}}}\right) \in O^{\times}
$$

Further from the proof of [Del73, Theorem 6.5(c)], it can be shown that $\varepsilon_{0}\left(V_{E}^{\sigma}\right)(\bmod \pi)$ depends on the residual representation $E[p] \otimes \widetilde{\sigma}$. In particular, $\varepsilon_{0}\left(V_{E_{1}}^{\sigma}\right)=\varepsilon_{0}\left(V_{E_{2}}^{\sigma}\right)(\bmod \pi)$.

Now, as $\rho_{E_{i} / F_{v}}^{\prime} \otimes \omega(v)^{1 / 2}$ is symplectic and $\sigma$ is self-dual, we can apply [Roh94, Lemma §12] to deduce $\left|\varepsilon\left(V_{E_{i}}^{\sigma}\right)\right|=\ell^{m_{i}}$. Further using $a\left(\rho_{E_{1} / F_{v}}^{\prime} \otimes \sigma\right)=a\left(\rho_{E_{2} / F_{v}}^{\prime} \otimes \sigma\right)$, we also deduce from [Roh94, Lemma §12], $m_{1}=m_{2}$ i.e. $\left|\varepsilon\left(V_{E_{1}}^{\sigma}\right)\right|=\left|\varepsilon\left(V_{E_{2}}^{\sigma}\right)\right|$.

Recall, $W\left(E_{i} / F_{v}, \sigma_{v}\right) \in\{ \pm 1\}$. As $\left|\varepsilon\left(V_{E_{i}}^{\sigma}\right)\right|=\ell^{m_{i}}$, using (32) and (33) we deduce that det (-Frob $\mid$ $\left.\left(V_{E_{i}}^{\sigma}\right)^{I_{F_{v}}}\right)$ is a $p$-adic unit. Hence, $\operatorname{det}\left(-\mathrm{Frob}_{v} \mid\left(V_{E_{i}}^{\sigma}\right)^{I_{F_{v}}}\right)(\bmod \pi)$ is an element of $\overline{\mathbb{F}}_{p}^{\times}$. Again since $\operatorname{Gal}\left(F_{\mathrm{cyc}, v} / F_{v}\right)$ is a pro- $p$ group, we obtain that

$$
\operatorname{det}\left(-\operatorname{Frob}_{v} \mid\left(V_{E_{i}}^{\sigma}\right)^{I_{F_{v}}}\right)=\operatorname{det}\left(-\operatorname{Frob}_{\mathrm{cyc}, v} \mid\left(V_{E_{i}}^{\sigma}\right)^{I_{F_{v}}}\right) \quad(\bmod \pi) .
$$

Using (34) in (32) and (33), we obtain the result.

With the notation of Theorem 2.18, put $m_{i}:=\sum_{v \in S_{i}}\left\langle\sigma_{v}, 1_{v}\right\rangle+\sum_{v \in N_{i}}\left\langle\sigma_{v}, \varkappa_{v}\right\rangle$ for $i=1,2$, and $T:=$ $\sum_{v \in W}\left(\left\langle\sigma_{v}, \vartheta_{v}\right\rangle+\left\langle\sigma_{v}, \omega_{v} \vartheta_{v}\right\rangle\right)+\sum_{v \in X}\left\langle\sigma_{v}, \vartheta_{v}\right\rangle+\sum_{v \in Y_{3}}\left(\left\langle\sigma_{v}, \varkappa_{v}\right\rangle+\left\langle\sigma_{v}, 1_{v}\right\rangle+\left\langle\sigma_{v}, \theta_{3, v}\right\rangle\right)+\sum_{v \in Z_{3}}\left(\left\langle\sigma_{v}, \vartheta_{v}\right\rangle+\right.$ $\left.\left\langle\sigma_{v}, \vartheta_{v} \theta_{3, v}\right\rangle\right)$. Then we have the following comparison of the root numbers of two congruent elliptic curves:

Theorem 3.4. Let $E_{1}, E_{2}$ be two elliptic curves over $k$ with good reductions at all primes of $k$ lying above an odd prime $p$ and $E_{1}[p] \cong E_{2}[p]$ as $G_{k}$-modules. Let $\sigma$ be an irreducible, self-dual, orthogonal representation of $\Delta$. Then

$$
\frac{W\left(E_{1} / F, \sigma\right)}{W\left(E_{2} / F, \sigma\right)}=(-1)^{m_{1}-m_{2}+T} .
$$

Proof. We will compare $W\left(E_{i} / F_{v}, \sigma_{v}\right)$ for $i=1,2$, as $v$ varies. By Theorem 3.1(i), we only need to consider finite primes. Further, if $E_{1}$ and $E_{2}$ have good reduction at $v$, then by Theorem 3.1(ii), $W\left(E_{1} / F_{v}, \sigma_{v}\right)=W\left(E_{2} / F_{v}, \sigma_{v}\right)$. In particular, this holds for all $v \mid p$. By Theorem 3.1(iii), the same conclusion holds if $E_{1}$ and $E_{2}$ are both split-multiplicative or both non-split multiplicative at $v$.

(i) (good - multiplicative). Suppose that $E_{1}$ has good reduction at $v$ and $E_{2}$ has multiplicative reduction at $v$. By Theorem 3.1, case (ii) and (iii), we deduce $\frac{W\left(E_{1} / F_{v}, \sigma_{v}\right)}{W\left(E_{2} / F_{v}, \sigma_{v}\right)}= \begin{cases}(-1)^{\left\langle\sigma_{v}, 1_{v}\right\rangle}, & \text { if } v \text { is split } \\ (-1)^{\left\langle\sigma_{v}, \varkappa_{v}\right\rangle}, & \text { if } v \text { is non-split. }\end{cases}$

(ii) (split - non-split). Assume that $E_{1}$ is split multiplicative at $v$ and $E_{2}$ is non-split multiplicative at $v$. By Theorem 3.1, we get $\frac{W\left(E_{1} / F_{v}, \sigma_{v}\right)}{W\left(E_{2} / F_{v}, \sigma_{v}\right)}=(-1)^{\left\langle\sigma_{v}, 1_{v}\right\rangle+\left\langle\sigma_{v}, \varkappa_{v}\right\rangle}$.

(iii) (multiplicative - additive)

(a) Let $E_{1}$ be multiplicative at $v$ and $E_{2}$ be PGA at $v$. From the proof of Lemma 2.15(ii), $p=3$, $3 \mid \# \rho_{E_{2}}\left(G_{F_{\mathrm{cyc}, v}}\right)$ and $\mu_{3} \subset F_{v}$. In this case, $\rho_{E_{2}, v} \cong \varepsilon_{E_{2}, v} \oplus \varepsilon_{E_{2}, v}^{-1}$. Since $\left.\rho_{E_{1}}\right|_{I_{F_{v}}} \sim\left(\begin{array}{cc}1 & * \\ 0 & 1\end{array}\right)$ and $\bar{\rho}_{E_{1}, v} \cong \bar{\rho}_{E_{2}, v}$, we deduce $\bar{\varepsilon}_{E_{2}, v}$ is unramified and thus $\bar{\varepsilon}_{E_{2}, v}(-1)=\varepsilon_{E_{2}, v}(-1)=1$. Then by Remark 3.2 and the cases (iii) \& (iv)(a) of Theorem 3.1, $\frac{W\left(E_{1} / F_{v}, \sigma_{v}\right)}{W\left(E_{2} / F_{v}, \sigma_{v}\right)}=(-1)^{\left\langle\sigma_{v}, 1_{v}\right\rangle}$ if $v$ is split and $=(-1)^{\left\langle\sigma_{v}, \varkappa_{v}\right\rangle}$ if $v$ is non-split. 
(b) Next we consider $E_{1}$ is multiplicative at $v$ and $E_{2}$ is PGNA at $v$. From the proof of Lemma 2.15(iii), this can only happen when $p=3,3 \mid \# \rho_{E_{2}}\left(G_{F_{\mathrm{cyc}, v}}\right), \mu_{3} \not \subset F_{v}$ and $e=\left.\# \rho_{E_{2}}\right|_{I_{F_{v}}}=3$. Moreover, $\rho_{E_{2}, v} \cong \theta_{3, v}$ is the unique 2-dim irreducible representation of $\operatorname{Gal}\left(F_{\text {cyc }, v}\left(E_{2}[p]\right) / F_{\text {cyc }, v}\right) \cong D_{6}$. Using Theorem 3.1(iv)(b), $\rho_{E_{2} / F_{v}} \otimes \omega(v)^{1 / 2}=\operatorname{Ind}_{L / F_{v}} \phi$ with $L / F_{v}$ is unramified quadratic and $W\left(E_{2} / F_{v}, \sigma_{v}\right)=\operatorname{det} \sigma_{v}(-1)(\phi(u))^{\operatorname{dim} \sigma}(-1)^{\left\langle\sigma_{v}, 1_{v}\right\rangle+\left\langle\sigma_{v}, \varkappa_{v}\right\rangle+\left\langle\sigma_{v}, \theta_{3, v}\right\rangle}$.

Note that the value of $\phi(u)$ is independent of $\sigma_{v}$. By [AAS17, Lemma 5.4], $\frac{W\left(E_{1} / F_{v}, 1_{v}\right)}{W\left(E_{2} / F_{v}, 1_{v}\right)}=1$ if $E_{1}$ is spilt at $v$ and -1 if $E_{1}$ is non-split at $v$. Therefore, we deduce that $\phi(u)$ appearing in the expression of $W\left(E_{2} / F_{v}, \sigma_{v}\right)$ above is equal to 1. Now using Theorem 3.1(iii), from the above discussion $\frac{W\left(E_{1} / F_{v}, \sigma_{v}\right)}{W\left(E_{2} / F_{v}, \sigma_{v}\right)}=(-1)^{\left\langle\sigma_{v}, \varkappa_{v}\right\rangle+\left\langle\sigma_{v}, \theta_{3, v}\right\rangle}$ if $E_{1}$ is split at $v$. Similarly, in the non-split case $\frac{W\left(E_{1} / F_{v}, \sigma_{v}\right)}{W\left(E_{2} / F_{v}, \sigma_{v}\right)}=(-1)^{\left\langle\sigma_{v}, 1_{v}\right\rangle+\left\langle\sigma_{v}, \theta_{3, v}\right\rangle}$.

(iv) (additive - additive).

(a) If both the curves are PMR at $v$, then by the proof of Lemma 2.16(i), either $\vartheta_{E_{1}, v}=$ $\vartheta_{E_{2}, v}$ in which case $W\left(E_{1} / F_{v}, \sigma_{v}\right)=W\left(E_{2} / F_{v}, \sigma_{v}\right)$. Otherwise, $\vartheta_{E_{1}, v}=\omega_{v} \vartheta_{E_{2}, v}$ and then, $\frac{W\left(E_{1} / F_{v}, \sigma_{v}\right)}{W\left(E_{2} / F_{v}, \sigma_{v}\right)}=(-1)^{\left\langle\sigma_{v}, \vartheta_{E_{1}, v}\right\rangle+\left\langle\sigma_{v}, \omega_{v} \vartheta_{E_{1}, v}\right\rangle}$.

(b) Next, assume $E_{1}$ is PMR at $v$ and $E_{2}$ is PGA at $v$. By Remark 3.2(1) and the cases (iii) and (iv) (a) of Theorem 3.1, we can express $\frac{W\left(E_{1} / F_{v}, \sigma_{v}\right)}{W\left(E_{2} / F_{v}, \sigma_{v}\right)}=\vartheta_{v}(-1)^{\operatorname{dim} \sigma}(-1)^{\left\langle\sigma_{v}, \vartheta_{E_{1}, v}\right\rangle} \varepsilon_{E_{2}, v}(-1)^{\operatorname{dim} \sigma}$. If $p \nmid \# \rho_{E_{2}}\left(G_{F_{\mathrm{cyc}, v}}\right)$, then using the proof of Lemma 2.16(iii), $\vartheta_{E_{1}, v} \in\left\{\varepsilon_{E_{2}, v}, \varepsilon_{E_{2}, v}^{-1} \omega_{v}\right\}$. As $\omega_{v}$ is unramified, $\frac{W\left(E_{1} / F_{v}, \sigma_{v}\right)}{W\left(E_{2} / F_{v}, \sigma_{v}\right)}=(-1)^{\left\langle\sigma_{v}, \vartheta_{E_{1}, v}\right\rangle}$.

Now if $p \mid \# \rho_{E_{2}}\left(G_{F_{\mathrm{cyc}, v}}\right)$, then as explained before, $p=3, \mu_{3} \subset F_{v}$ and $\omega_{v}=1_{v}$. Thus $\rho_{E_{1}, v} \sim\left(\begin{array}{cc}\vartheta_{E_{1}, v} & * \\ 0 & \vartheta_{E_{1}, v}\end{array}\right)$ and $\rho_{E_{2}, v} \cong \varepsilon_{E_{2}, v} \oplus \varepsilon_{E_{2}, v}^{-1}$. As $\bar{\rho}_{E_{1}, v} \cong \bar{\rho}_{E_{2}, v}$, we conclude that $\vartheta_{E_{1}, v}(-1)=\varepsilon_{E_{2}, v}(-1)$. Then using Remark 3.2 and Theorem 3.1 (iii) \& (iv)(a), $\frac{W\left(E_{1} / F_{v}, \sigma_{v}\right)}{W\left(E_{2} / F_{v}, \sigma_{v}\right)}=$ $(-1)^{\left\langle\sigma_{v}, \vartheta_{E_{1}, v}\right\rangle}$.

(c) Let $E_{1}$ be PMR at $v$ and $E_{2}$ be PGNA at $v$. Then $p=3, \mu_{3} \not \subset F_{v}$ and $3 \mid \# \rho_{E_{2}}\left(G_{F_{\mathrm{cyc}, v}}\right)$ (see Lemma 2.16(ii)). Then $E_{1}^{\prime}:=E_{1}^{\vartheta_{E_{1}, v}}$ is split at $v$. Recall, $\rho_{E_{2}, v} \cong \theta_{6, v}$, a certain 2-dim. irreducible representation of the dihedral group of order 12. Also, $E_{2}^{\prime}:=E_{2}^{\vartheta_{E_{1}, v}}$ is PGNA at $v$. Note that $E_{1}^{\prime}[3] \cong E_{2}^{\prime}[3]$. Then by a similar argument, as in the proof of Lemma 2.16(ii), we get $\left.\# \bar{\rho}_{E_{2}^{\prime}}\right|_{I_{F_{v}}}=\left.\# \rho_{E_{2}^{\prime}}\right|_{I_{F_{v}}}=3$. Therefore, $\rho_{E_{2}^{\prime}, v} \cong \theta_{3, v}$, that is, $\rho_{E_{2}, v} \otimes \vartheta_{E_{1}, v} \cong \theta_{6, v} \otimes \vartheta_{E_{1}, v} \cong \theta_{3, v}$.

Now using part (iii)(b) of this proposition related to the (split) multiplicative - additive case, we obtain $\frac{W\left(E_{1}^{\prime} / F_{v}, \sigma_{v} \vartheta_{E_{1}, v}\right)}{W\left(E_{2}^{\prime} / F_{v}, \sigma_{v} \vartheta_{E_{1}, v}\right)}=(-1)^{\left\langle\sigma_{v} \vartheta_{E_{1}, v}, \varkappa_{v}\right\rangle+\left\langle\sigma_{v} \vartheta_{E_{1}, v}, \theta_{3, v}\right\rangle}$. Notice that $\vartheta_{E_{1}, v}$ being quadratic (self dual), $W\left(E_{i}^{\prime} / F_{v}, \sigma_{v} \vartheta_{E_{1}, v}\right)=W\left(E_{i} / F_{v}, \sigma_{v}\right)$ for $i=1,2$ and also $\left\langle\sigma_{v}, \vartheta_{E_{1}, v} \chi\right\rangle=\left\langle\sigma_{v} \vartheta_{E_{1}, v}, \chi\right\rangle$ for $\chi \in\left\{\vartheta_{E_{1}, v}, \varkappa_{v}\right\}$. Moreover, as $p=3$ and $\mu_{3} \notin F_{v}$, the unramified quadratic character $\varkappa_{v}=\omega_{v}$ and hence $\left\langle\sigma_{v}, \varkappa_{v} \vartheta_{E_{1}, v}\right\rangle=\left\langle\sigma_{v}, \omega_{v} \vartheta_{E_{1}, v}\right\rangle$. Hence, we deduce $\frac{W\left(E_{1} / F_{v}, \sigma_{v}\right)}{W\left(E_{2} / F_{v}, \sigma_{v}\right)}=(-1)^{\left\langle\sigma_{v}, \omega_{v} \vartheta_{E_{1}, v}\right\rangle+\left\langle\sigma_{v}, \theta_{3, v} \vartheta_{E_{1}, v}\right\rangle}$.

(d) Let $E_{1}, E_{2}$ both have PGA at $v$. Recall, $\rho_{E_{i}, v}$ is a representation of $\operatorname{Gal}\left(F_{\text {cyc }, v}\left(E_{i}[p]\right) / F_{\text {cyc }, v}\right)$ for $i=1,2$ (see §2). Using [Ser72, Page 312], $\rho_{E_{i}}\left(I_{F_{v}}\right) \cong \bar{\rho}_{E_{i}}\left(I_{F_{v}}\right)$ is cyclic of order $e$ with possible value of $e \in\{2,3,4,6\}$. Moreover, $\bar{\rho}_{E_{1}, v} \cong \bar{\rho}_{E_{2}, v}$ implies that $e=\left.\# \rho_{E_{1}}\right|_{I_{F_{v}}}=$ $\left.\# \rho_{E_{2}}\right|_{I_{F_{v}}}$. Then for $i=1,2$, there is either one faithful representation $\varepsilon_{E_{i}, v}$, or two faithful representations $\varepsilon_{E_{i}, v}, \varepsilon_{E_{i}, v}^{-1}$ of the inertia subgroup of $\operatorname{Gal}\left(F_{\mathrm{cyc}, v}\left(E_{i}[p]\right) / F_{\mathrm{cyc}, v}\right)$. In either 
cases, we deduce $\varepsilon_{E_{1}, v}(-1)=\varepsilon_{E_{2}, v}(-1)$. Now by Remark 3.2(1) and Theorem 3.1(iv)(a), the local root numbers at $v$ are the same.

(e) Next, assume $E_{1}, E_{2}$ both are PGNA at $v$. In this case, we have $\rho_{E_{i} / F_{v}}^{\prime}=\rho_{E_{i} / F_{v}}$ for $i=1,2$. As observed in Lemma 2.16(vi), we also have $\rho_{E_{1}, v} \cong \rho_{E_{2}, v}$. Note that $I_{F_{v}} \subset G_{F_{\mathrm{cyc}, v}}$ and $a\left(\rho_{E_{i} / F_{v}} \otimes \sigma\right)$ just depends on the restriction of $\rho_{E_{i} / F_{v}} \otimes \sigma$ to the inertia subgroup. Hence using the fact $\rho_{E_{1}, v} \cong \rho_{E_{2}, v}$, we obtain $a\left(\rho_{E_{1} / F_{v}} \otimes \sigma\right)=a\left(\rho_{E_{2} / F_{v}} \otimes \sigma\right)$. Now by Lemma 3.3, we conclude $W\left(E_{1} / F_{v}, \sigma_{v}\right)=W\left(E_{2} / F_{v}, \sigma_{v}\right)$.

By Lemmas $2.7-2.16$, these are the only possibilities of reduction types for the pair $E_{1}, E_{2}$. Thus from the above discussion, the result follows.

3.3. The relation between multiplicities and root numbers. Using Theorem 2.18, Remark 2.20 and Theorem 3.4, we obtain the main result of this article:

Theorem 3.5. Let $p$ be an odd prime and $k$ be a number field such that all primes of $k$ lying above $p$ are ramified in $k\left(\mu_{p}\right) / k$. Let $E_{1}, E_{2}$ be elliptic curves over $k$ with $E_{1}[p] \cong E_{2}[p]$ as $G_{k}$-modules. Let $\sigma: \Delta \rightarrow \mathrm{GL}_{n}\left(\overline{\mathbb{Q}}_{p}\right)$ be an irreducible self-dual orthogonal representation. Assume the hypothesis $(\mathbf{H})$. Then

$$
(-1)^{s_{p}\left(E_{1}, \sigma\right)-s_{p}\left(E_{2}, \sigma\right)}=\frac{W\left(E_{1} / F, \sigma\right)}{W\left(E_{2} / F, \sigma\right)} .
$$

In particular, the p-parity conjecture in (2) holds for the twist of $E_{1} / F$ by $\sigma$ if and only if it holds for the twist of $E_{2} / F$ by $\sigma$.

Remark 3.6. [Necessity of the condition (H)] The hypothesis (H4) stating $S_{p}\left(E_{i}\left[p^{\infty}\right] / K\right)[p]$ is finite, is necessary in the proof of Theorem 2.4, in Lemma 1.1 and also in (12). In fact, all the 4 hypothesis (H1) - (H4) was used in the proof of Theorem 2.4. The hypothesis (H1) is also used in the proof of Theorem 3.4 .

Remark 3.7. [ $\sigma$ symplectic] We have assumed $\sigma$ to be orthogonal in our results. In the other case, when $\sigma$ is (irreducible, self dual and) symplectic, then assuming non-degeneracy of the height pairing, the equality $(-1)^{s_{p}\left(E_{1}, \sigma\right)-s_{p}\left(E_{2}, \sigma\right)}=\frac{W\left(E_{1} / F, \sigma\right)}{W\left(E_{2} / F, \sigma\right)}$ is trivially true, by the following results of Greenberg and Rohrlich:

[Gre11, Prop. 10.2.3] Let $E / \mathbb{Q}$ be good ordinary at $p$. Assume that the $p$-adic height pairing on $S_{p}(E / K)^{\vee} \otimes_{\mathbb{Z}_{p}} \mathbb{Q}_{p}$ is non-degenerate and $S_{p}\left(E / K_{\text {cyc }}\right)^{\vee}$ is a finitely generated torsion $\Lambda=\mathbb{Z}_{p}\left[\left[\mathrm{Gal}\left(K_{\mathrm{cyc}} / K\right)\right]\right]$ module. Let $\sigma$ be a self-dual, irreducible representation of $\Delta$. Then $s_{p}(E, \sigma) \equiv \lambda_{E}(\sigma)(\bmod 2)$. Moreover, if $\sigma$ is assumed to be symplectic, then $\lambda_{E}(\sigma)$ is even.

Under the setting of the above paragraph, when $\sigma$ is symplectic, by [Roh96, Proposition 2], we get the global root number $W(E / F, \sigma)=1$. Thus an analogues of Theorem 3.5 is easily obtained.

Remark 3.8 (The reverse situation). It may be natural to change the setting by fixing one elliptic curve $E$ and consider two self dual representation $\rho_{1}, \rho_{2}: \Delta \longrightarrow \operatorname{GL}_{n}(\mathcal{F})$, such that $\tilde{\rho}_{1} \cong \tilde{\rho}_{2}$, where $\tilde{\rho}_{i}$ is as defined in $\S 1$. In this setting, the comparison of the $p$-parity conjecture for twist of $E$ by $\rho_{1}$ with that of $E$ by $\rho_{2}$, has been already established by Greenberg; see [Gre11, Theorem 3] for a precise statement. 
3.4. Examples: We now discuss numerical examples to demonstrate application of our results. Note that the computation over a number field $\neq \mathbb{Q}$ (for example, $p$-descent for $p \neq 2$ ) is difficult and hence it is not easy to find explicit numerical examples. We thank V. Dokchitser for several helpful suggestions.

Example 3.9. Let $E_{1}, E_{2}$ be the two elliptic curves from LMFDB:

$$
\begin{gathered}
E_{1}=11 . a 2: y^{2}+y=x^{3}-x^{2}-7820 x-263580, \\
E_{2}=737 . a 1: y^{2}+y=x^{3}-x^{2}+406 x-686 .
\end{gathered}
$$

Take $p=3$ and $k=F=\mathbb{Q}$. Note the dimension of $S_{2}\left(\Gamma_{0}(11)\right)=1$ and $737=11 \cdot 67$, by level lowering (or using SAGE), we can conclude $E_{1}[3] \cong E_{2}[3]$ as $G_{\mathbb{Q}}$-modules.

Now $E_{1}$ has good reduction at 3 and from LMFDB, $a_{3}\left(f_{E_{1}}\right)=-1$, where $f_{E_{1}}$ is the newform of weight 2 attached to $E_{1}$ via modularity. Thus $E_{1}$ is 3-ordinary.

Let $K=10.10 .1559914552888693 .1$ (marking as in LMFDB) be the totally real number field of degree 10 over $\mathbb{Q}$ with discriminant $K=1093^{5}$, which is Galois over $\mathbb{Q}$ with $\Delta:=\operatorname{Gal}(K / \mathbb{Q}) \cong D_{5}$, the dihedral group of order 10. The ideal class group of $K$ is trivial. Note that $\Delta$ has two 2-dimensional self dual, orthogonal, irreducible (faithful) representations and we denote by $\sigma$ any one of them.

We check, via Magma, that $L\left(E_{1} / K, 1\right) \neq 0$. As $K$ is totally real and the analytic rank of $E_{1}$ over $K$ is 0 , we can apply the BSD over $K$ ([Zha01, Theorem A]) to conclude, $E_{1}(K)$ is finite and $\amalg\left(E_{1} / K\right)$ is also finite. Thus $S_{3}\left(E_{1} / K\right)$ is finite and consequently $s_{3}\left(E_{1}, \sigma\right)=0$. On the other hand, $E_{1}$ has bad reduction only at $q=11$ and $\sigma$ is unramified at 11. Hence using [Roh96, Proposition 10], we deduce $W\left(E_{1} / \mathbb{Q}, \sigma\right)=1$. So the 3-parity conjecture holds for $\left(E_{1}, \mathbb{Q}, \sigma\right)$.

Using SAGE, we see that $E_{1}(K)[3]=0$ and in fact, $E_{1}(K)[3]$ is an irreducible $G_{K}$ module. Moreover, as $S_{3}\left(E_{1} / K\right)$ is finite, using a control theorem, we deduce $S_{3}\left(E_{1} / K_{\text {cyc }}\right)^{\vee}$ is a torsion $\mathbb{Z}_{3}[[\Gamma]]$ module. Thus all the condition of our theorem, except that the Iwasawa $\mu$-invariant of $S_{3}\left(E_{1} / K_{\text {cyc }}\right)^{\vee}$ i.e. $\mu\left(S_{3}\left(E_{1} / K_{\text {cyc }}\right)^{\vee}\right) \stackrel{?}{=} 0$, are satisfied. We also observe in SAGE that the Tamagawa numbers of $E_{1}$ over $K$ are 3 -adic units. Further, $3 \nmid \# \tilde{E}_{1}(\kappa)$ as well, where $\kappa$ is the residue field of $K$ at a prime dividing 3 .

Recall, $\# \amalg_{a n}$ is defined to be the conjectural order of the $\amalg$, as predicted by the exact formula appearing in the strong form of the BSD conjecture. Using Magma, we compute that $3 \nmid \# \amalg_{a n}\left(E_{1} / K\right)$. Note that in our setting, strong form of the BSD conjecture is not established yet over the totally real field $K$; even for elliptic curves with analytic rank $\leq 1$. Also, over $K$, a totally real Galois field of degree 10 over $\mathbb{Q}$, the one half of the Iwasawa Main Conjecture (extension of Kato's divisibility) has not been proven yet. Assuming $3 \nmid \# \amalg_{a n}\left(E_{1} / K\right) \Longrightarrow 3 \nmid \# \amalg\left(E_{1} / K\right)$, we have $\amalg\left(E_{1} / K\right)[3]=0$. Hence we get that $S_{3}\left(E_{1} / K\right)=0$ and then using control theorem and Nakayama's lemma, we can deduce that $S_{3}\left(E_{1} / K_{\text {cyc }}\right)=0$.

Then $\left(E_{1}, E_{2}, \mathbb{Q}, \sigma\right)$ satisfy all the conditions of Theorem 3.5 and applying the same theorem, we deduce the 3 -parity conjecture holds for $\left(E_{2}, \mathbb{Q}, \sigma\right)$. We check via SAGE, $W\left(E_{2} / \mathbb{Q}, \sigma\right)=1$. Thus, we conclude from Theorem 3.5 that $s_{3}\left(E_{2}, \sigma\right) \equiv 0(\bmod 2)$.

On the other hand, we have calculated via Magma, $\operatorname{ord}_{s=1} L\left(E_{2} / K, s\right) \geq 2$. As $3 \nmid \# D_{5}$, we can not deduce the 3-parity conjecture for $\left(E_{2}, \mathbb{Q}, \sigma\right)$ using regulator constants or existing results in the literature (cf. [DD09], [DD11]). We summarize the example below. (Also see Remark 3.13.) 
Corollary 3.10. Let $E_{1}, E_{2}, K$ be as above. Assume that $3 \nmid \# \amalg\left(E_{1} / K\right)_{a n} \Longrightarrow 3 \nmid \# \amalg\left(E_{1} / K\right)$. Then 3-parity conjecture holds for $\left(E_{2}, \mathbb{Q}, \sigma\right)$ with $W\left(E_{2} / \mathbb{Q}, \sigma\right)=1$ and $s_{3}\left(E_{2}, \sigma\right) \equiv 0(\bmod 2)$.

Example 3.11. Let $E_{1}, E_{2}$ be the two elliptic curves as considered in [GV00, Page 22]:

$$
\begin{gathered}
E_{1}=52 . a 1: y^{2}+y=x^{3}+x-10, \\
E_{2}=364 . a 1: y^{2}+y=x^{3}-584 x+5444 .
\end{gathered}
$$

Take $p=5$ and $k=F=\mathbb{Q}$. It is shown in [GV00] that $E_{1}[5] \cong E_{2}[5]$ as $G_{\mathbb{Q}}$-modules and $E_{1}$ has good, ordinary reduction at 5 .

Let $K=6.6 .16974593 .1$ (marking as in LMFDB) be the totally real number field of degree 6 with discriminant $K=257^{3}$, which is Galois over $\mathbb{Q}$ with $\Delta:=\operatorname{Gal}(K / \mathbb{Q}) \cong S_{3}$. The ideal class group of $K$ is trivial. Note that $\Delta$ has a 2-dimensional self dual, orthogonal, irreducible representation, say $\sigma$.

We check, via Magma, that $L\left(E_{1} / K, 1\right) \neq 0$. As $K$ is totally real and the analytic rank of $E_{1}$ over $K$ is 0 , we can apply the BSD over $K$ ([Zha01, Theorem A]) to conclude, $E_{1}(K)$ is finite and $\amalg\left(E_{1} / K\right)$ is also finite. Thus $S_{5}\left(E_{1} / K\right)$ is finite and consequently $s_{5}\left(E_{1}, \sigma\right)=0$. On the other hand, $E_{1}$ has bad reduction at $q=2,13$ and $\sigma$ is unramified at 13 and 2. Hence again using [Roh96, Proposition 10], we deduce $W\left(E_{1} / \mathbb{Q}, \sigma\right)=1$. So the 5 -parity conjecture for the twist of $E_{1}$ by $\sigma$ over $\mathbb{Q}$ holds true.

Using SAGE, we see that $E_{1}(K)[5]=0$ and in fact, $E_{1}(K)[5]$ is an irreducible $G_{K}$ module. Moreover, as $S_{5}\left(E_{1} / K\right)$ is finite, via a control theorem, we deduce $S_{5}\left(E_{1} / K_{\text {cyc }}\right)^{\vee}$ is a torsion $\mathbb{Z}_{5}[[\Gamma]]$ module. Thus all the condition of our theorem, except $\mu\left(S_{5}\left(E_{1} / K_{\text {cyc }}\right)^{\vee}\right) \stackrel{?}{=} 0$, are satisfied. We also observe in SAGE that the Tamagawa numbers of $E_{1}$ over $K$ are 5 -adic units. Further, $5 \nmid \# \tilde{E}_{1}(\kappa)$, where $\kappa$ is the residue field of $K$ at a prime dividing 5 .

Using Magma, we have computed that $5 \nmid \# \amalg_{a n}\left(E_{1} / K\right)$. Again, the strong form of BSD conjecture or the Iwasawa Main Conjecture over $K$ has not been proven yet. Assuming $5 \nmid \# \amalg_{a n}\left(E_{1} / K\right) \Longrightarrow 5 \nmid$ $\# \amalg\left(E_{1} / K\right)$, we have $\amalg\left(E_{1} / K\right)[5]=0$. Thus, we obtain $S_{5}\left(E_{1} / K\right)=0$ and as in the previous example, using control theorem and Nakayama's lemma, we deduce $S_{5}\left(E_{1} / K_{\text {cyc }}\right)=0$.

Then $\left(E_{1}, E_{2}, \mathbb{Q}, \sigma\right)$ satisfy all the conditions of Theorem 3.5 and applying the same theorem, we deduce the 5-parity conjecture holds for $\left(E_{2}, \mathbb{Q}, \sigma\right)$. Notice that, again applying [Roh96, Proposition 10], we see that $W\left(E_{2} / \mathbb{Q}, \sigma\right)=-1$. Thus by Theorem 3.5 , we get $s_{5}\left(E_{2} / \mathbb{Q}, \sigma\right) \equiv 1(\bmod 2)$.

On the other hand, we have calculated via Magma, $\operatorname{ord}_{s=1} L\left(E_{2} / K, s\right)=2$. As $5 \nmid \# S_{3}$, we can not deduce the 5-parity conjecture for $\left(E_{2}, \mathbb{Q}, \sigma\right)$ using regulator constants or existing results in the literature (cf. [DD09], [DD11]). Thus we have shown:

Corollary 3.12. Let $E_{1}, E_{2}, K$ be as above. Assume that $5 \nmid \# \amalg\left(E_{1} / K\right)_{a n} \Longrightarrow 5 \nmid \# \amalg\left(E_{1} / K\right)$. Then 5 -parity conjecture holds for $\left(E_{2}, \mathbb{Q}, \sigma\right)$ with $W\left(E_{2} / \mathbb{Q}, \sigma\right)=-1$ and $s_{5}\left(E_{2}, \sigma\right) \equiv 1(\bmod 2)$.

Remark 3.13. We stress that the condition $p \nmid \# \amalg(E / K)_{a n} \Longrightarrow p \nmid \# \amalg(E / K)$ in Examples 3.9 and 3.11 is only used to check the vanishing of $\mu\left(S_{p}\left(E / K_{\text {cyc }}\right)^{\vee}\right)$. According to the Iwasawa main conjecture for $E$ over a totally real field $K$, the characteristic ideal of $S_{p}\left(E / K_{\mathrm{cyc}}\right)^{\vee}$ is given by certain $p$-adic $L$ function of $E$ over $K$, say $\mathcal{L}_{p}(E / K)$, which depends on the choice of certain canonical period. In the above two examples, from the $p$-adic BSD formula, one has $\mathcal{L}_{p}(E / K)$ is a unit in the Iwasawa algebra 
$\Lambda$. Let $\mu_{a n}$ be defined by $p^{\mu_{a n}} \| \mathcal{L}_{p}(E / K)$ in $\Lambda$. Hence the above condition in Corollaries 3.10 and 3.12 involving $\# \amalg(E / K)_{a n}$ and \#Ш $\left.E / K\right)$ can be replaced by the following condition:

$$
\mu_{\text {an }}=0 \Longrightarrow \mu\left(S_{p}\left(E / K_{\text {cyc }}\right)^{\vee}\right)=0 .
$$

Of course, one half of the Iwasawa main conjecture of $E$ over $K_{\text {cyc }}$ stating the characteristic ideal $\operatorname{char}_{\Lambda}\left(S_{p}\left(E / K_{\text {cyc }}\right)^{\vee}\right) \mid \mathcal{L}_{p}(E / K)$ is proved, then the condition in (35) will be satisfied.

There are recent works of [Lo12], [Wa15], where they have shown in the setting of Hilbert modular forms of parallel weight, the characteristic ideal of Selmer group over anticyclotomic $\mathbb{Z}_{p}$ extension divides the corresponding anticylotomic $p$-adic $L$-function. The condition $p \nmid \# \amalg(E / K)_{a n} \Longrightarrow p \nmid \# \amalg(E / K)$ can be deduced from this divisibility. However, these works are conditional on a form of Ihara's Lemma. The paper on arXiv [Xi19] has removed this dependence on Ihara's Lemma. The relation between $\# \amalg(E / \mathbb{Q})_{a n}$ and $\# \amalg(E / \mathbb{Q})$ is also discussed in [BHT17].

In any case, as $E_{i}[p]$ is an irreducible $G_{K}$-module, it is always expected that the $\mu\left(S_{p}\left(E_{i} / K_{\text {cyc }}\right)^{\vee}\right)=0$ (see discussions following [Gre11, Theorem 1]).

Remark 3.14. By results of Rubin-Silverberg [RS93], given an elliptic curve $E_{1} / \mathbb{Q}$ and for $p \in\{3,5\}$, there are infinitely many elliptic curves $E / \mathbb{Q}$ such that $E[p] \cong E_{1}[p]$ as $G_{\mathbb{Q}}$ modules. Thus, under the assumption on \#Ш $\left(E_{1} / K\right)[p]$, Example 3.9 (respectively Example 3.11) shows, there are infinitely many elliptic curves $E / \mathbb{Q}$ such that the 3-parity (respectively 5-parity) conjecture is true for each of $(E, K, \sigma)$. In fact, there are similar phenomenon of congruences for elliptic curves for prime $p=7,11,13$ (cf. [Fis14]).

Now, we discuss an example where all of our assumptions in Theorem 3.5 (including $\mu=0$ ) are (unconditionally) satisfied.

Example 3.15. Let $E_{1}, E_{2}$ be the two elliptic curves from LMFDB:

$$
\begin{gathered}
E_{1}=56 . b 1: y^{2}=x^{3}-x^{2}-4, \\
E_{2}=392 . c 1: y^{2}=x^{3}-x^{2}-16 x+29 .
\end{gathered}
$$

Take $p=3$ and $k=\mathbb{Q}$. Note that $j\left(E_{1}\right)=-2^{2} \cdot 7^{-1}$. Using SAGE, we check that $E_{1}[3] \cong E_{2}[3]$ as $G_{\mathbb{Q}^{-}}$ modules. Let $\zeta_{19}$ denote a primitive 19-th roots of unity. Set $F:=\mathbb{Q}\left(\zeta_{19}+\zeta_{19}^{-1}\right)$ and let $K:=F\left(\mu_{3}, m^{1 / 3}\right)$, where $m$ is any 3-power free integer. Let $\sigma$ be an irreducible, self dual, orthogonal representation of $\Delta=\operatorname{Gal}(K / F) \cong S_{3}$.

We will now show that all the conditions of Theorem 3.5 are satisfied for $\left(E_{1}, E_{2}, F, \sigma\right)$ and we will compute the parity of $s_{p}\left(E_{1}, \sigma\right)-s_{p}\left(E_{2}, \sigma\right)$ using our results.

We check from LMFDB that $E_{1}$ is 3-ordinary. Now $\mathbb{Q}\left(\mu_{3}\right)_{\text {cyc }}=\mathbb{Q}\left(\mu_{3^{\infty}}\right)$ being abelian, $S_{3}\left(E_{1} / \mathbb{Q}\left(\mu_{3} \infty\right)\right)^{\vee}$ is $\Lambda$-torsion by results of Kato. Also, from [DD07, Table B.1] we notice that the $\mu\left(S_{3}\left(E_{1} / \mathbb{Q}\left(\mu_{3^{\infty}}\right)^{\vee}\right)=0\right.$. As, $\left[K_{\text {cyc }}: \mathbb{Q}\left(\mu_{3 \infty}\right)\right]$ is a power of 3 , the $\mu$-invariant of $E_{1}$ over $K_{\text {cyc }}$ also vanishes by [HM99, Corollary $3.4])$. Further, from the same table we have $E_{1}\left(\mathbb{Q}\left(\mu_{3}\right)\right)[3]=0$. As $\left[K: \mathbb{Q}\left(\mu_{3}\right)\right]=27$, we get $E_{1}(K)[3]=0$ as well. Then all the conditions of Theorem 3.5 are satisfied.

The 3-parity conjecture for $\left(E_{1}, F, \sigma\right)$ holds by [DD11, Theorem 6.13]. Then applying Theorem 3.5, we can deduce 3 -parity for $\left(E_{2}, F, \sigma\right)$. However, as $\Delta \cong S_{3}$ and $p=3$, the 3-parity conjecture for both 
$\left(E_{1}, F, \sigma\right)$ and $\left(E_{2}, F, \sigma\right)$ also follows from [DD11, Example 6.10], using the results of [DD11] on regulator constants. We mention that the $p$-parity conjecture for the twist of $E_{2} / F$ by $\sigma$ does not follow from [DD11, Theorem 6.13] or [DD09, Theorem 1.3].

This discussion also shows that the 3-parity conjecture holds for any elliptic curve $E / F$ twisted by $\sigma$ that satisfies $E[3] \cong E_{1}[3]$ as $G_{\mathbb{Q}}$-modules.

Now we determine $s_{3}\left(E_{1}, \sigma\right)-s_{3}\left(E_{2}, \sigma\right)(\bmod 2)$. First, note that the primes $v$ of $F$ dividing 7 belong to $\Sigma_{0}$ and for such a $v$, we have $\delta_{E_{1}, v}-\delta_{E_{2}, v} \equiv\left\langle\sigma_{v}, 1_{v}\right\rangle(\bmod 2)$ by Lemma 2.15 (ii). For a prime $v$ of $F$ dividing $2, E_{1}, E_{2}$ have potentially good, additive reduction. By Lemma 2.13 , for $K=F\left(\mu_{3}, m^{1 / 3}\right)$, a prime of $F$ lying above 2, belongs to $\Sigma_{0}$ if and only if $m$ is even. When $v$ in $F$ dividing 2 is in $\Sigma_{0}$, by Lemma 2.16, parts (iv), (v) and (vi), $\delta_{E_{1}, v}-\delta_{E_{2}, v} \equiv 0(\bmod 2)$. Thus, from the table or Theorem 2.18, we deduce $s_{3}\left(E_{1}, \sigma\right)-s_{3}\left(E_{2}, \sigma\right) \equiv \sum_{v \mid 7 \text { in } F}\left\langle\sigma_{v}, 1_{v}\right\rangle(\bmod 2)$. Let us take $u|v| 7$ where $u, v$ respectively are primes in $K$ and $F$. Since $\mu_{3} \subset F_{v}$, we see that $\operatorname{Gal}\left(K_{u} / F_{v}\right)$ is trivial or has order 3. If $K_{u}=F_{v}$, then $\left\langle\sigma_{v}, 1_{v}\right\rangle=2$. On the other hand if $\left[K_{u}: F_{v}\right]=3$, it is easy to see that $\left\langle\sigma_{v}, 1_{v}\right\rangle=0$.

Thus from the above discussion, for any $K=F\left(\mu_{3}, m^{1 / 3}\right)$, we always get $s_{3}\left(E_{1}, \sigma\right) \equiv s_{3}\left(E_{2}, \sigma\right)$ $(\bmod 2)$. Similarly, we can deduce $W\left(E_{1} / F, \sigma\right) \equiv W\left(E_{2} / F, \sigma\right)$.

\section{RELATion With the theORY of 'ARIthmetic local CONSTANTS'}

Mazur-Rubin have developed the theory of 'arithmetic local constants' in [MR07] which has been used by Nekovár [Ne15]. We explain the relation of our method with the theory of arithmetic local constants. For simplicity, we consider in this section the special case where $F=K$ and hence $\sigma$ is trivial. However, we remark about the general case with $\sigma$ non-trivial at the end.

Let $E_{1}, E_{2}$ be two elliptic curves over a number field $F$. We say $E_{1}[p] \cong E_{2}[p]$ as symplectic $G_{F}$ modules if the $G_{F}$ linear isomorphism $E_{1}[p] \cong E_{2}[p]$ is compatible with the natural, symplectic Weil pairing on $E_{i}[p]$. Throughout this section, we keep the following hypothesis:

(Syl): $E_{1}[p] \cong E_{2}[p]$ as symplectic $G_{F}$ modules.

Under the Hypothesis (Syl), using Mazur-Rubin's theory of arithmetic local constants, Nekovár [Ne15] has expressed the both (i) the ratio of Selmer corank of $E_{1}, E_{2}$ and (ii) the ratio of the local root numbers of $E_{1}$ and $E_{2}$ at a finite prime $v \nmid p$ of $F$ in terms of the arithmetic local constant $d\left(\mathcal{F}_{v}^{1}, \mathcal{F}_{v}^{2}\right)$. To state his results more precisely, we need the following definitions.

For a finite prime $v \nmid p$ of $F$, let $I_{v}$ denote the inertia subgroup of $G_{F_{v}}$ at $v$. Note that there is a natural inclusion map from $T_{p} E_{i} \longrightarrow V_{p} E_{i}:=T_{p} E_{i} \otimes \mathbb{Q}_{p}$ and a natural quotient map $T_{p} E_{i} \longrightarrow \frac{T_{p} E_{i}}{p T_{p} E_{i}} \cong E_{i}[p]$. These map induce corresponding maps on cohomology. Put (see [Ne15, §2])

$H_{f}^{1}\left(T_{p} E_{i} / F_{v}\right):=\operatorname{ker}\left(H^{1}\left(F_{v}, T_{p} E_{i}\right) \rightarrow \frac{H^{1}\left(F_{v}, V_{p} E_{i}\right)}{H^{1}\left(G_{F_{v}} / I_{v}, V_{p} E_{i}^{I_{v}}\right)}\right)$ and $\mathcal{F}_{v}^{i}:=\operatorname{Image}\left(H_{f}^{1}\left(T_{p} E_{i} / F_{v}\right) \rightarrow H^{1}\left(F_{v}, E_{i}[p]\right)\right)$.

Then $\mathcal{F}_{v}^{i}$ is a finite dimensional $\mathbb{F}_{p}$ vector space and is a Lagrangian subspace of $H^{1}\left(F_{v}, E_{1}[p]\right) \cong$ $H^{1}\left(F_{v}, E_{2}[p]\right)$. For a prime $v \mid p$ of $F$, we omit the definition of $\mathcal{F}_{v}^{i}$ (see [Ne15, §3.2]). Given any two Lagrangian subspaces $\mathcal{F}_{v}^{1}, \mathcal{F}_{v}^{2}$ at a finite prime $v$ of $F$, define the arithmetic local constant,

$$
d\left(\mathcal{F}_{v}^{1}, \mathcal{F}_{v}^{2}\right):=\operatorname{dim}_{\mathbb{F}_{p}} \frac{\mathcal{F}_{v}^{1}}{\mathcal{F}_{v}^{1} \cap \mathcal{F}_{v}^{2}} \quad(\bmod 2)=\operatorname{dim}_{\mathbb{F}_{p}} \frac{\mathcal{F}_{v}^{2}}{\mathcal{F}_{v}^{1} \cap \mathcal{F}_{v}^{2}} \quad(\bmod 2) \in \mathbb{Z} / 2 \mathbb{Z}
$$


Recall the following exact sequence induced by Kummer theory via the multiplication by $p$ map on $E_{i}$ :

$$
0 \longrightarrow \frac{E_{i}\left(F_{v}\right)}{p E_{i}\left(F_{v}\right)} \stackrel{\kappa_{v}^{i}}{\longrightarrow} H^{1}\left(F_{v}, E_{i}[p]\right) \longrightarrow H^{1}\left(F_{v}, E_{i}\left[p^{\infty}\right]\right)[p] \longrightarrow 0
$$

For elliptic curves, at $v \nmid p, H^{1}\left(G_{F_{v}} / I_{v}, V_{p} E_{i}^{I_{v}}\right)$ always vanishes. Using this, it is easy to see that

$$
\mathcal{F}_{v}^{i}=\operatorname{Image}\left(\kappa_{v}^{i}\right) \cong \frac{E_{i}\left(F_{v}\right)}{p E_{i}\left(F_{v}\right)} .
$$

Assume the condition (Syl) holds. Then the setting of [Ne15, §2.16] applies and we deduce from [Ne15, Theorem 2.17] that for a finite prime $v \nmid p$ of $F$,

$$
\frac{W\left(E_{1} / F_{v}\right)}{W\left(E_{2} / F_{v}\right)}=(-1)^{d\left(\mathcal{F}_{v}^{1}, \mathcal{F}_{v}^{2}\right)}
$$

On the other hand, assume (Syl) and in addition, also assume $E_{i}[p](F)=0$. Then it follows from [MR07, Theorem 1.4], [Ne15, Equ. (4.6.2)] that

$$
s_{p}\left(E_{1}\right)-s_{p}\left(E_{2}\right)=\sum_{v \mid p N_{E_{1}}^{F} N_{E_{2}}^{F}} d\left(\mathcal{F}_{v}^{1}, \mathcal{F}_{v}^{2}\right) \quad(\bmod 2) .
$$

Remark 4.1. We stress that we do not assume this (Syl) hypothesis in Theorem 3.5. Even if $E_{1}$ and $E_{2}$ are sympletically isomorphic i.e. they satisfy (Syl), the analogue of (40) at a prime $v \mid p$ of $F$ is not clear and the situation is more complicated (see [Ne15, §3] and his subsequent works).

When $\sigma$ is trivial, we denote $\delta_{E_{i}, v}:=\delta_{E_{i}, v}(\sigma)$, where $\delta_{E, v}(\sigma)$ is defined in $\S 1$. In Theorem 3.4, we have expressed the ratio of local root numbers $\frac{W\left(E_{1} / F_{v}\right)}{W\left(E_{2} / F_{v}\right)}$ in terms of $\delta_{E_{1}, v}-\delta_{E_{2}, v}(\bmod 2)$. On the other hand, Nekovár have expressed (in (40)) the same ratio $\frac{W\left(E_{1} / F_{v}\right)}{W\left(E_{2} / F_{v}\right)}, v \nmid p$ in terms of $d\left(\mathcal{F}_{v}^{1}, \mathcal{F}_{v}^{2}\right)$. Now we will show directly in Proposition 4.3, without invoking any results of Nekovar or of this article, that under (Syl), at $v \nmid p$ of $F$, the difference of our local Iwasawa invariant $\delta_{E_{1}, v}-\delta_{E_{2}, v}=d\left(\mathcal{F}_{v}^{1}, \mathcal{F}_{v}^{2}\right)(\bmod 2)$. This 'completes the triangle' and shows that our methods are compatible with that of Nekovár. However to prove Proposition 4.3, we first need the following lemma:

Lemma 4.2. Let $E$ be an elliptic curve over $F$ and $v$ be a finite prime of $F$ such that $v \nmid p$. Then the natural map $\frac{E\left(F_{v}\right)}{p E\left(F_{v}\right)} \longrightarrow \frac{E\left(F_{\mathrm{cyc}, v}\right)}{p E\left(F_{\mathrm{cyc}, v}\right)}$ is surjective.

Proof. We prove this by considering the reduction type of $E$ at $v$. If $E$ has good reduction at $v \nmid p$, then $E\left[p^{\infty}\right]\left(F_{\mathrm{cyc}, v}\right)$ is $p$-divisible and hence $\frac{E\left(F_{\mathrm{cyc}, v}\right)}{p E\left(F_{\mathrm{cyc}, v}\right)} \cong \frac{E\left[p^{\infty}\right]\left(F_{\mathrm{cyc}, v}\right)}{p E\left[p^{\infty}\right]\left(F_{\mathrm{cyc}, v}\right)}=0$ and we get the required surjectivity trivially. Next, if $E$ has additive reduction at $v$ with $p \geq 5$, then consider $F_{v}\left(\mu_{p}\right)$, an unramified extension of $F_{v}$. Then $E$ continues to be additive over $F_{v}\left(\mu_{p}\right)$ and by [HM99, Prop. 5.1(iii)], $E\left[p^{\infty}\right]\left(F_{v}\left(\mu_{p^{\infty}}\right)\right)=0$ and hence $E\left[p^{\infty}\right]\left(F_{\mathrm{cyc}, v}\right)=0$. On the other hand, if $\mu_{p} \subset F_{v}$ and $E$ has non-split multiplicative reduction at $v$, then $p$ being odd, $E$ continues to have non-split multiplicative reduction over $F_{\mathrm{cyc}, v}$ and again by [HM99, Prop. 5.1(iii)], we get $E\left[p^{\infty}\right]\left(F_{\text {cyc }, v}\right)=0$.

Let $F_{v}=F_{v_{0}} \subset F_{v_{n}} \ldots \subset F_{\text {cyc }, w}$ be the layers of the (unramified) cyclotomic $\mathbb{Z}_{p}$ extension of $F$ with $\operatorname{Gal}\left(F_{v_{n}} / F_{v}\right) \cong \mathbb{Z} / p^{n} \mathbb{Z}$. To deal with the remaining cases, we make a couple of observations, to be used. First, as $\frac{E\left(F_{\mathrm{cyc}, v}\right)}{p E\left(F_{\mathrm{cyc}, v}\right)}$ is finite, the natural map from $\frac{E\left(F_{v_{n}}\right)}{p E\left(F_{v_{n}}\right)} \longrightarrow \frac{E\left(F_{\mathrm{cyc}, v}\right)}{p E\left(F_{\mathrm{cyc}, v}\right)}$ is surjective for some sufficiently large $n$. Thus the required surjectivity in the lemma will follow if we show the surjectivity of $\frac{E\left(F_{v}\right)}{p E\left(F_{v}\right)} \stackrel{b_{E}}{\longrightarrow}$ $\frac{E\left(F_{v_{n}}\right)}{p E\left(F_{v_{n}}\right)}$ for any $n$. Secondly, via a diagram chase using the multiplication by $p$ map, respectively on $E\left(F_{v}\right)$ 
and $E\left(F_{v_{n}}\right)$, we observe that the map $b_{E}$ is surjective if and only if $\operatorname{dim}_{\mathbb{F}_{p}} E[p]\left(F_{v}\right)=\operatorname{dim}_{\mathbb{F}_{p}} E[p]\left(F_{v_{n}}\right)$. In particular, if $E[p]\left(F_{v}\right)=0$ then by Nakayama's Lemma, $E[p]\left(F_{v_{n}}\right)=0$ as well. Thus we only need to consider the cases when $E[p]\left(F_{v}\right) \neq 0$.

If $E$ has additive reduction over $F_{v}$ then $E[p]\left(F_{v}\right) \neq 0$ can happen only when $p=3$. So we consider $p=3$ and $E$ has additive reduction at $v$. Then recall $E$ attains good reduction over $F_{v}(E[3])$ [ST68, Corollaries 2(b) \& 3]. Hence using $F_{v_{n}} / F_{v}$ is unramified, we conclude that $\operatorname{dim}_{\mathbb{F}_{p}} E[p](L)=2$ is not possible for $L \in\left\{F_{v}, F_{v_{n}}\right\}$. Hence if $\operatorname{dim}_{\mathbb{F}_{p}} E[p]\left(F_{v}\right)=1$ then $\operatorname{dim}_{\mathbb{F}_{p}} E[p]\left(F_{v_{n}}\right)=1$ as well.

Next, we assume that $\mu_{p} \not \subset F_{v}$ and $E$ has split (respectively non-split) reduction at $v$. Then recall from $\S 1$, the diagonal entries of $\left.\rho_{E}\right|_{G_{F_{v}}}$ are $\left\{\omega_{v}, 1\right\}$ (respectively $\left\{\omega_{v} \varkappa_{v}, \varkappa_{v}\right\}$; where $\varkappa_{v}$ is a unramified quadratic character). Keeping in mind that $F_{v_{n}} / F_{v}$ is a $p$-power extension, $\left.\chi\right|_{G_{F v}}=\left.1 \Leftrightarrow \chi\right|_{G_{F_{n}}}=1$, where $\chi$ represents any of the above diagonal characters. Thus we see, $\operatorname{dim}_{\mathbb{F}_{p}} E[p]\left(F_{v_{n}}\right) \leq 1$ and it is the same as $\operatorname{dim}_{\mathbb{F}_{p}} E[p]\left(F_{v}\right)$.

Finally, we consider the case where $\mu_{p} \subset F_{v}$ and $E$ is split-multiplicative at $v$. Recall $(\S 1),\left.\rho_{E}\right|_{G_{F_{v}}} \sim$ $\left(\begin{array}{ll}1 & * \\ 0 & 1\end{array}\right)$. If $* \neq 0$ for $\left.\rho_{E}\right|_{G_{F v}}$ but $*$ becomes 0 for $\left.\rho_{E}\right|_{G_{F_{n}}}$ then some $p$-power root of the Tate parameter $q$ ([HM99, Prop 5.1(ii)]) does not belong to $F_{v}$ but it is in $F_{v_{n}}$. However, then $F_{v_{n}} / F_{v}$ will be a ramified Kummer extension, which is a contradiction. Thus $* \neq 0$ over $F_{v}$ if and only if $* \neq 0$ over $F_{v_{n}}$. Hence if $\operatorname{dim}_{\mathbb{F}_{p}} E[p]\left(F_{v}\right)=1$ (when $\left.* \neq 0\right)$, then $\operatorname{dim}_{\mathbb{F}_{p}} E[p]\left(F_{v_{n}}\right)=1$ also. Similarly, when the former rank is 2 (when $*=0$ ) then latter rank is 2 as well. This completes the proof of the lemma.

Proposition 4.3. Assume the hypothesis (Syl) stated in this section. Then for a finite prime $v \nmid p$ of $F$,

$$
\delta_{E_{1}, v}-\delta_{E_{2}, v}=d\left(\mathcal{F}_{v}^{1}, \mathcal{F}_{v}^{2}\right)(\bmod 2)
$$

Proof. We identify the isomorphic $G_{F}$ modules $E_{1}[p]$ and $E_{2}[p]$ in this proposition and denote both of them as $E[p]$ in this proof. Recall the unramified cohomology $\mathcal{F}_{v}^{u n}:=H^{1}\left(G_{F_{v}} / I_{v},(E[p])^{I_{v}}\right)$ is a Lagrangian subspaces of $H^{1}\left(G_{F_{v}}, E[p]\right)$ [Ne15, §2]. Then further recall, by a result of Klagsbrun-MazurRubin (see [Ne15, Prop. 2.9]),

$$
d\left(\mathcal{F}_{v}^{1}, \mathcal{F}_{v}^{2}\right)=d\left(\mathcal{F}_{v}^{u n}, \mathcal{F}_{v}^{1}\right)-d\left(\mathcal{F}_{v}^{u n}, \mathcal{F}_{v}^{2}\right)=\operatorname{dim}_{\mathbb{F}_{p}} \frac{\mathcal{F}_{v}^{1}}{\mathcal{F}_{v}^{u n} \cap \mathcal{F}_{v}^{1}}-\operatorname{dim}_{\mathbb{F}_{p}} \frac{\mathcal{F}_{v}^{2}}{\mathcal{F}_{v}^{u n} \cap \mathcal{F}_{v}^{2}} \quad(\bmod 2)
$$

We choose a fix a prime $w$ in $F_{\text {cyc }}$ dividing the prime $v$ in $F$. As our calculations below does not depend on the choice of $w$ above $v$, by abuse of notation, we will denote $F_{\text {cyc }, w}$ by $F_{\text {cyc }, v}$. By Kummer theory, we have an exact sequence

$$
0 \longrightarrow \frac{E_{i}\left(F_{\mathrm{cyc}, v}\right)}{p E_{i}\left(F_{\mathrm{cyc}, v}\right)} \stackrel{\kappa_{\mathrm{cyc}}^{i} v}{\longrightarrow} H^{1}\left(F_{\mathrm{cyc}, v}, E_{i}[p]\right) \longrightarrow H^{1}\left(F_{\mathrm{cyc}, v}, E_{i}\left[p^{\infty}\right]\right)[p] \longrightarrow 0
$$

Further, by well known results of Greenberg (cf. [Gre11]), $H^{1}\left(F_{\mathrm{cyc}, v}, E_{i}[p]\right)$ is finite and as $v \nmid p$, $\delta_{E_{i}, v}=s_{v} \times \operatorname{dim}_{\mathbb{F}_{p}} H^{1}\left(F_{\mathrm{cyc}, v}, E_{i}\left[p^{\infty}\right]\right)[p]$, where $s_{v}$ is the number of primes above $v$ in $F_{\text {cyc }}$ (see discussion above (14)). Thus, it is immediate that

$$
\delta_{E_{1}, v}-\delta_{E_{2}, v}=\operatorname{dim}_{\mathbb{F}_{p}} \frac{E_{1}\left(F_{\mathrm{cyc}, v}\right)}{p E_{1}\left(F_{\mathrm{cyc}, v}\right)}-\operatorname{dim}_{\mathbb{F}_{p}} \frac{E_{2}\left(F_{\mathrm{cyc}, v}\right)}{p E_{2}\left(F_{\mathrm{cyc}, v}\right)} \quad(\bmod 2) .
$$


Now we have the commutative diagram:

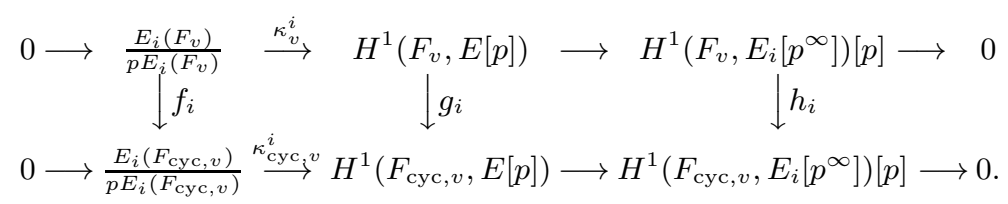

By Lemma 4.2, $f_{i}$ is surjective in (46), for $i=1,2$. So we deduce from (45), $\delta_{E_{1}, v}-\delta_{E_{2}, v}=$ $\operatorname{dim}_{\mathbb{F}_{p}} \operatorname{Img}\left(f_{1}\right)-\operatorname{dim}_{\mathbb{F}_{p}} \operatorname{Img}\left(f_{2}\right)(\bmod 2)$, where $\operatorname{Img}\left(f_{i}\right)$ stands for the Image $\left(f_{i}\right)$. Moreover, using (39), we can express

$$
\delta_{E_{1}, v}-\delta_{E_{2}, v}=\operatorname{dim}_{\mathbb{F}_{p}} \mathcal{F}_{v}^{1}-\operatorname{dim}_{\mathbb{F}_{p}} \mathcal{F}_{v}^{2}-\left(\operatorname{dim}_{\mathbb{F}_{p}} \operatorname{ker}\left(f_{1}\right)-\operatorname{dim}_{\mathbb{F}_{p}} \operatorname{ker}\left(f_{1}\right)\right) \quad(\bmod 2) .
$$

Further, we have $\operatorname{Gal}\left(F_{v}^{\mathrm{unr}} / F_{\text {cyc }, v}\right) \cong \prod_{\ell \neq p} \mathbb{Z}_{\ell}$. Hence we deduce that the natural restriction map from $H^{1}\left(F_{\text {cyc }, v}, E[p]\right) \rightarrow H^{1}\left(I_{v}, E[p]\right)$ is injective. Consequently, the kernel of $g_{i}$ is $\mathcal{F}_{v}^{u n}$. Now, notice that

$$
\operatorname{dim}_{\mathbb{F}_{p}} \operatorname{ker}\left(f_{i}\right)=\operatorname{dim}_{\mathbb{F}_{p}} \kappa_{v}^{i}\left(\operatorname{ker}\left(f_{i}\right)\right)=\operatorname{dim}_{\mathbb{F}_{p}}\left(\operatorname{ker}\left(g_{i}\right) \cap \operatorname{Img}\left(\kappa_{v}^{i}\right)\right)=\operatorname{dim}_{\mathbb{F}_{p}}\left(\mathcal{F}_{v}^{u n} \cap \mathcal{F}_{v}^{i}\right) .
$$

Applying (47) and (48) in the expression of $d\left(\mathcal{F}_{v}^{1}, \mathcal{F}_{v}^{2}\right)$ in (43), it is immediate that

$$
\delta_{E_{1}, v}-\delta_{E_{2}, v}=d\left(\mathcal{F}_{v}^{1}, \mathcal{F}_{v}^{2}\right) \quad(\bmod 2) .
$$

This completes the proof of the proposition.

Remark 4.4. In the general case, when $F \neq K$ and $\sigma$ non-trivial, irreducible, self dual representation of $\operatorname{Gal}(K / F)$, then note that if (Syl) is satisfied i.e. $E_{1}[p] \cong E_{2}[p]$ as symplectic $G_{F}$-modules, then $E_{1}[p] \otimes \widetilde{\sigma} \cong E_{2}[p] \otimes \widetilde{\sigma}$ also symplectically as $G_{F}$-modules. Thus in a similar way to Proposition 4.3, $\delta_{E_{1}, v}(\sigma)-\delta_{E_{2}, v}(\sigma)(\bmod 2)$ can be expressed in terms of arithmetic local constants.

Acknowledgements: S. Jha is supported by SERB ECR and SERB MATRICS grant. T. Mandal acknowledges institute postdoctoral fellowship at IIT Kanpur. S. Shekhar is supported by DST INSPIRE faculty grant. The numerical computations were done using SAGE and LMFDB. We thank Vladimir Dokchister for various discussions.

\section{REFERENCES}

[AAS17] Suman Ahmed, Chandrakant Aribam, and Sudhanshu Shekhar. Root numbers and parity of local Iwasawa invariants. J. Number Theory, 177:285-306, 2017.

[BHT17] Ashay Burungale, Haruzo Hida and Ye Tian, Horizontal variation of Tate-Shafarevich groups preprint, arXiv:1712.02148v2.

[DD09] Tim Dokchitser and Vladimir Dokchitser. Regulator constants and the parity conjecture. Invent. Math., 178(1):23-71, 2009.

[DD11] Tim Dokchitser and Vladimir Dokchitser. Root numbers and parity of ranks of elliptic curves. J. Reine Angew. Math., 658:39-64, 2011.

[DD07] T. Dokchitser and V. Dokchitser. Computations in non-commutative Iwasawa theory. Proc. Lond. Math. Soc. (3), 94(1):211-272, 2007. With an appendix by J. Coates and R. Sujatha.

[DDT94] Henri Darmon, Fred Diamond, and Richard Taylor. Fermat's last theorem. In Current developments in mathematics, 1995 (Cambridge, MA), pages 1-154. 
[Del73] P. Deligne. Les constantes des équations fonctionnelles des fonctions $L$. pages 501-597. Lecture Notes in Math., Vol. 349, 1973.

[EPW06] Matthew Emerton, Robert Pollack, and Tom Weston. Variation of Iwasawa invariants in Hida families. Invent. Math., 163(3):523-580, 2006.

[Fis14] T. Fisher, On families of 7- and 11-congruent elliptic curves, LMS J. Comput. Math. 17 (2014), no. $1,536-564$.

[Gre10] Ralph Greenberg. Selmer groups and congruences. In Proceedings of the International Congress of Mathematicians. Volume II, pages 231-248. Hindustan Book Agency, New Delhi, 2010.

[Gre11] Ralph Greenberg. Iwasawa theory, projective modules, and modular representations. Mem. Amer. Math. Soc., 211(992):vi+185, 2011.

[GV00] R. Greenberg and V. Vatsal, On the Iwasawa invariants of elliptic curves, Invent. Math. 142 (2000), 17-63.

[HM99] Yoshitaka Hachimori and Kazuo Matsuno. An analogue of Kida's formula for the Selmer groups of elliptic curves. J. Algebraic Geom., 8(3):581-601, 1999.

[HV03] Yoshitaka Hachimori and Otmar Venjakob. Completely faithful Selmer groups over Kummer extensions. Extra Vol. of Documenta Math., 443-478. 2003. Kazuya Kato's fiftieth birthday.

[Li89] R. Livné, On the conductors of mod 1 Galois representations coming from modular forms, $J$. Number Theory 31 (1989), no. 2, 133-141.

[LS18] Meng Fai Lim and Ramdorai Sujatha. Fine Selmer groups of congruent Galois representations. J. Number Theory, 187:66-91, 2018.

[Lo12] M. Longo, Anticyclotomic Iwasawa main conjecture for Hilbert modular forms, Comment. Math. Helv. 87 (2012), 303-353.

[MR07] B. Mazur \& K. Rubin, Finding large Selmer rank via an arithmetic theory of local constants. Annals of Mathematics, 166, 579-612, 2007.

[Ne15] J. Nekovarr, Compatibility of arithmetic and algebraic local constants (the case $\ell \neq p$ ), Compositio Math. 151, 1626-1646, 2015.

[Roh94] David E. Rohrlich. Elliptic curves and the Weil-Deligne group. In Elliptic curves and related topics, CRM Proc. Lecture Notes, 125-157 (4). Amer. Math. Soc., Providence, 1994.

[Roh96] David E. Rohrlich. Galois theory, elliptic curves, and root numbers. Compositio Math., 100(3):311-349, 1996.

[Roh08] David E. Rohrlich. Scarcity and abundance of trivial zeros in division towers. J. Algebraic Geom., 17(4):643-675, 2008.

[RS93] K. Rubin and A. Silverberg, Families of elliptic curves with constant mod $p$-representations, Elliptic curves, modular forms, \& Fermat?s last theorem, Hong Kong, 1993.

[Sil94] Joseph H. Silverman. Advanced topics in the arithmetic of elliptic curves, volume 151 of Graduate Texts in Mathematics. Springer-Verlag, New York, 1994.

[She16] Sudhanshu Shekhar. Parity of ranks of elliptic curves with equivalent $\bmod p$ Galois representations. Proc. Amer. Math. Soc., 144(8):3255-3266, 2016.

[Zha01] Shou-Wu Zhang, Heights of Heegner points on Shimura curves, Ann. of Math. (2), 153(1): 27-147, 2001. 
[Ser72] Jean-Pierre Serre. Propriétés galoisiennes des points d'ordre fini des courbes elliptiques. Invent. Math., 15(4):259-331, 1972.

[ST68] Jean-Pierre Serre and John Tate. Good reduction of abelian varieties. Ann. of Math. (2), 88:492-517, 1968.

[Xi19] Bingyong Xie, Iwasawa Theory of Hilbert modular forms for anticyclotomic extension, arXiv:1909.10162.

[Wa15] Haining Wang, Anticyclotomic Iwasawa Theory for Hilbert modular forms. PhD thesis, The Penn. State University, 2015, arXiv1909.12374.

[Wei03] Steven H. Weintraub. Representation theory of finite groups: algebra and arithmetic, volume 59 of Graduate Studies in Mathematics. American Mathematical Society, Providence, 2003.

Somnath Jha, Jhasom@ittk.ac.in, IIT Kanpur \& IIT Goa, India

Tathagata Mandal, math.tathagata@gmail.com, IIT Kanpur, India

Sudhanshu Shekhar, sudhansh@ittk.AC.In, IIT Kanpur, India 